\title{
Generalizing the entanglement entropy of singular regions in conformal field theories
}

\author{
Pablo Bueno, ${ }^{a}$ Horacio Casini $^{a}$ and William Witczak-Krempa ${ }^{b, c}$ \\ ${ }^{a}$ Instituto Balseiro, Centro Atómico Bariloche, \\ 8400-S.C. de Bariloche, Río Negro, Argentina \\ ${ }^{b}$ Departement de physique, Université de Montréal, \\ Montréal, Québec, H3C 3J\%, Canada \\ ${ }^{c}$ Centre de Recherches Mathématiques, Université de Montréal, \\ P.O. Box 6128, Centre-ville Station, Montréal, Québec, H3C 3J\%, Canada \\ E-mail: pablo.bueno@cab.cnea.gov.ar, casini@cab.cnea.gov.ar, \\ w.witczak-krempa@umontreal.ca
}

ABSTRACT: We study the structure of divergences and universal terms of the entanglement and Rényi entropies for singular regions. First, we show that for $(3+1)$-dimensional free conformal field theories (CFTs), entangling regions emanating from vertices give rise to a universal contribution $S_{n}^{\text {univ }}=-\frac{1}{8 \pi} f_{b}(n) \int_{\gamma} k^{2} \log ^{2}(R / \delta)$, where $\gamma$ is the curve formed by the intersection of the entangling surface with a unit sphere centered at the vertex, and $k$ the trace of its extrinsic curvature. While for circular and elliptic cones this term reproduces the general-CFT result, it vanishes for polyhedral corners. For those, we argue that the universal contribution, which is logarithmic, is not controlled by a local integral, but rather it depends on details of the CFT in a complicated way. We also study the angle dependence for the entanglement entropy of wedge singularities in $3+1$ dimensions. This is done for general CFTs in the smooth limit, and using free and holographic CFTs at generic angles. In the latter case, we show that the wedge contribution is not proportional to the entanglement entropy of a corner region in the $(2+1)$-dimensional holographic CFT. Finally, we show that the mutual information of two regions that touch at a point is not necessarily divergent, as long as the contact is through a sufficiently sharp corner. Similarly, we provide examples of singular entangling regions which do not modify the structure of divergences of the entanglement entropy compared with smooth surfaces.

KEYwords: Conformal Field Theory, AdS-CFT Correspondence

ARXIV EPRINT: 1904.11495 


\section{Contents}

1 Introduction 1

1.1 Summary of results 4

2 Vertex-induced universal terms $\quad 5$

2.1 Dimensional reduction for free fields 6

2.2 Polyhedral corners 11

2.2.1 Cubes in the extensive mutual information model 12

$\begin{array}{lll}2.3 & \text { Elliptic cones } & 14\end{array}$

$\begin{array}{lll}2.3 .1 & \text { General CFTs } & 14\end{array}$

2.3.2 Free fields 20

2.4 Higher dimensions $\quad 21$

3 Wedge entanglement versus corner entanglement $\quad 21$

3.1 General CFTs in the nearly smooth limit 22

3.2 Free fields 23

$\begin{array}{lll}3.3 & \text { Holography } & 25\end{array}$

4 Singular geometries versus entanglement divergences $\quad 27$

4.1 Finite mutual information for touching regions 28

$\begin{array}{ll}4.2 & \text { Entanglement entropy of curved corners }\end{array}$

5 Conclusion $\quad 34$

A Cone entanglement in the Extensive Mutual Information model 35

B Which cone maximizes the Rényi entropy? 36

C Hyperconical entanglement in even dimensions $\quad 37$

\section{Introduction}

Given a bipartition of the Hilbert space of a quantum system $\mathcal{H}=\mathcal{H}_{V} \otimes \mathcal{H}_{\bar{V}}$, the Rényi and entanglement entropies associated with $V$ in some state $\rho$ are defined as

$$
S_{n}(V)=\frac{1}{1-n} \log \operatorname{Tr} \rho_{V}^{n}, \quad S_{\mathrm{EE}}(V)=\lim _{n \rightarrow 1} S_{n}(V)=-\operatorname{Tr}\left(\rho_{V} \log \rho_{V}\right),
$$

where $\rho_{V}=\operatorname{Tr}_{\bar{V}} \rho$ is the partial-trace density matrix obtained by integrating the degrees of freedom in the complement of $V$. In the context of quantum field theory, Rényi entropies 
are intrinsically UV-divergent. ${ }^{1}$ In particular, given a smooth entangling region $V$ on a time slice of a $d$-dimensional conformal field theory (CFT), the Rényi entropy takes the generic form — see e.g., [3, 4],

$$
S_{n}^{(d)}=b_{d-2} \frac{H^{d-2}}{\delta^{d-2}}+b_{d-4} \frac{H^{d-4}}{\delta^{d-4}}+\cdots+ \begin{cases}b_{1} \frac{H}{\delta}+(-1)^{\frac{d-1}{2}} s_{n}^{\text {univ }}, & (\text { odd } d), \\ b_{2} \frac{H^{2}}{\delta^{2}}+(-1)^{\frac{d-2}{2}} s_{n}^{\text {univ }} \log \left(\frac{H}{\delta}\right)+b_{0}, & (\text { even } d) .\end{cases}
$$

In this expression, $H$ is some characteristic length of $V, \delta$ is a UV regulator, and the (Rényi index-dependent) coefficients $b_{i}$ are non-universal, i.e. they depend on the regularization scheme.

In even dimensions, the universal term is logarithmic and its coefficient, $s_{n}^{\text {univ }}$, is controlled by a linear combination of local integrals on the entangling surface $\partial V \equiv \Sigma$ weighted by certain theory-dependent charges which reduce to the corresponding trace-anomaly coefficients for $n=1$. The simplest case corresponds to a segment of length $H$ in a twodimensional CFT, for which $[5,6] S_{n}^{(2)}=\frac{c}{6}\left(1+\frac{1}{n}\right) \log \left(\frac{H}{\delta}\right)+\mathcal{O}\left(\delta^{0}\right)$, where $c$ is the Virasoro central charge. The next case is that of four-dimensional theories. For those, there are three theory-dependent functions of the Rényi index, customarily denoted $f_{a}(n), f_{b}(n)$ and $f_{c}(n)$, which control the linear combination of local integrals characterizing the logarithmic universal contribution $[7,8]$ - see eq. (2.42) below. For $n=1$, they reduce to the usual trace-anomaly coefficients, $f_{a}(1)=a, f_{b}(1)=f_{c}(1)=c$. An analogous story holds for $d \geq 6$ - see e.g., $[9,10]$. The situation is different in odd dimensions though. For those, no logarithmic contribution is present for smooth entangling surfaces, and the universal contribution is a constant term which no longer corresponds to a simple local integral over $\Sigma$. The simplest case corresponds to three-dimensional CFTs, for which ${ }^{2} S_{n}^{(3)}=b_{1} \frac{H}{\delta}-F_{n}$. For $\Sigma=\mathbb{S}^{1}, F$ actually equals the free energy of the corresponding theory on $\mathbb{S}^{3}[13,14]$, which reveals its non-local nature. ${ }^{3}$

When geometric singularities are present in $\Sigma$, the structure of divergences in eq. (1.2) gets modified. The prototypical case is that of an entangling region bounded by a corner of opening angle $\Omega$ for three-dimensional CFTs. In that case, a new logarithmic universal contribution appears

$$
S_{n}^{(3) \text { corner }}=b_{1} \frac{H}{\delta}-a_{n}^{(3)}(\Omega) \log \left(\frac{H}{\delta}\right)+b_{0},
$$

where $a_{n}^{(3)}(\Omega)$ is a cutoff-independent function of the opening angle which has been extensively studied in the literature - e.g., for free fields in [15-22], for large- $N$ vector models in [23], for holographic theories in [24-39], in interacting lattice models in [40-45], and for general CFTs in [46-50].

\footnotetext{
${ }^{1}$ See [1] and [2] for two interesting recent reviews with somewhat complementary scopes.

${ }^{2}$ Constant terms such as $F_{n}$ are less robust than their even-dimensional logarithmic counterparts. This is because we cannot resolve the relevant IR scales of the entangling region with more precision than the UV cutoff. If we shift the relevant characteristic scale as $R \rightarrow R+a \delta$, with $a=\mathcal{O}(1)$, we will pollute the putative universal contribution as $F_{n} \rightarrow F_{n}\left(1-b_{1} a\right)$. This pollution - which does not occur for logarithmic contributions - can be remedied using mutual information as a geometric regulator [11, 12].

${ }^{3}$ Note that when we omit the subindex $n$ from the different contributions, we will be referring to the entanglement entropy case, corresponding to $n=1$.
} 
The explicit dependence of $a_{n}^{(3)}(\Omega)$ on the opening angle and the Rényi index changes from one CFT to another - e.g., compare the relatively simple holographic result [24] with the highly complicated resulting expressions for free fields [15-17]. The nature of $a_{n}^{(3)}(\Omega)$ is in stark contrast with that of the analogous coefficient corresponding to a conical entangling surface in four-dimensions. In that case, a similar logarithmic enhancement of the universal term does occur, and the Rényi entropy reads

$$
S_{n}^{(4) \text { cone }}=b_{2} \frac{H^{2}}{\delta^{2}}-a_{n}^{(4)}(\Omega) \log ^{2}\left(\frac{H}{\delta}\right)+b_{0} \log \left(\frac{H}{\delta}\right)+\mathcal{O}\left(\delta^{0}\right) .
$$

For the cone, however, the universal function $a_{n}^{(4)}(\Omega)$ is much more constrained than $a_{n}^{(3)}(\Omega)$. On the one hand, the dependence on the opening angle and the Rényi index factorize and, on the other, the explicit angular dependence is the same for all four-dimensional CFTs, namely $^{4}[25,57]$

$$
a_{n}^{(4)}(\Omega)=\frac{1}{4} f_{b}(n) \frac{\cos ^{2} \Omega}{\sin \Omega} .
$$

This contrast can be understood from the fact that both $a_{n}^{(3)}(\Omega)$ and $a_{n}^{(4)}(\Omega)$ can be thought of as emerging from the respective contributions $s_{n}^{\text {univ }}$ in eq. (1.2) - see e.g., [52]. While in $d=3$ this is a constant and non-local term, in $d=4$ it is a geometric integral over the entangling surface. As we explain here, the origin of this difference can be made extremely manifest in the case of free fields, for which the computation of $a_{n}^{(3)}(\Omega)$ requires the full evaluation of a spectral function on $\mathbb{S}^{2}$ with a cut of angle $\Omega$ while $a_{n}^{(4)}(\Omega)$ arises from a simple local integral on the curve resulting from the intersection of the cone with a unit $\mathbb{S}^{2}$ centered at its tip - see our summary of results below.

There are other interesting singular regions one can think of in $d=4$. For example, we can consider the case of a polyhedral corner of opening angles $\theta_{1}, \theta_{2}, \ldots, \theta_{j}$. In particular, this geometry is natural in lattice simulations. In that case, it has been observed that the universal contribution is not quadratically logarithmic, but just logarithmic, like in the case of smooth entangling regions, namely [58-62]

$$
S_{n}^{(4) \text { polyhedral }}=b_{2} \frac{H^{2}}{\delta^{2}}-w_{1} \frac{H}{\delta}+v_{n}\left(\theta_{1}, \theta_{2}, \cdots, \theta_{j}\right) \log \left(\frac{L}{\delta}\right)+\mathcal{O}\left(\delta^{0}\right) .
$$

In $[60,61]$, the idea that $v_{n}\left(\theta_{1}, \theta_{2}, \cdots, \theta_{j}\right)$ might be controlled by a simple linear combination of the functions $f_{a}(n)$ and $f_{b}(n)$ was put forward, although a definite conclusion was not reached. Here, we will use free-field calculations to understand the true origin of $v_{n}\left(\theta_{1}, \theta_{2}, \cdots, \theta_{j}\right)$, and rule out this possibility.

In eq. (1.6) we observe the appearance of an additional nonuniversal contribution weighted by some constant $w_{1}$. While $v_{n}\left(\theta_{1}, \theta_{2}, \cdots, \theta_{j}\right)$ comes from the corner itself, $w_{1}$

\footnotetext{
${ }^{4}$ Observe that a remarkable degree of universality was nonetheless shown to hold for $a^{(3)}(\Omega)$ in $[26,46,47]$, in the sense that normalizing this function by the stress-tensor two-point function charge $C_{T}$ [51], the curves corresponding to very different theories become very close to each other. The agreement becomes exact in the almost smooth limit, namely, $a^{(3)}(\Omega)=\frac{\pi^{2}}{24} C_{T}(\pi-\Omega)^{2}+\ldots$ for general CFTs. The result generalizes to higher-dimensional (hyper)cones $[28,52]-$ see also [53]. The possible generalization to $n \neq 1$ Rényi entropies turns out to be trickier [18-20, 32, 50, 54-56].
} 
arises from the presence of wedges in the entangling region. If we consider a simpler setup corresponding to an infinitely extended wedge region of opening angle $\Omega$, the Rényi entropy is in turn given by $[25,57]$

$$
S_{n}^{(4) \text { wedge }}=b_{2} \frac{H^{2}}{\delta^{2}}-f_{n}(\Omega) \frac{H}{\delta}+\mathcal{O}\left(\delta^{0}\right),
$$

where $f_{n}(\Omega)$ is a function of the wedge opening angle. Naturally, the overall normalization cannot be well defined, since simple redefinitions of the cutoff modify it. However, using holographic and free-field calculations, it has been suggested [57] that the angular dependence of $f_{n}(\Omega)$ matches the one corresponding to a corner region in one dimension less, namely $\partial_{\Omega}\left(f_{n}(\Omega) / a_{n}^{(3)}(\Omega)\right) \stackrel{(?)}{=} 0$. We present a careful study of the relation between both functions that reveals that the angular dependence of $f_{n}(\Omega)$ differs from $a_{n}^{(3)}(\Omega)$ in general.

In all cases mentioned so far, the entangling surfaces can be thought of as families of straight lines emanating from a vertex. However, one can consider more general entangling surfaces, such as the ones arising from curved corners. In the second part of the paper we will study the somewhat surprising interplay between those and the structure of divergences and universal terms in the Rényi entropy and also the mutual information. A detailed summary of our results can be found next.

\subsection{Summary of results}

$\diamond$ In section 2, we consider the problem of universal contributions to the Rényi entropy induced by the presence of vertices in the entangling region. In particular, for a $d$ dimensional free scalar field, we show that the usual logarithmic contribution can be related to the Rényi entropy of a region with the same angular boundary conditions on $(d-1)$-dimensional de Sitter space. Performing a high-mass expansion of the latter, we show that the logarithmic contribution gets enhanced to a quadratically logarithmic term of the form

$$
\left.S_{n}\right|_{\log ^{2}}=\frac{-f_{b}(n)}{8 \pi} \log ^{2} \delta \int_{\gamma} k^{2},
$$

where $\gamma$ is the curve resulting from the intersection of the entangling region $V$ with the unit $\mathbb{S}^{2}$ centered at its tip, and $k$ the trace of the extrinsic curvature of $\gamma$. In the case of cones and elliptic cones, we explicitly verify that this term agrees with the result obtained from Solodukhin's formula. For polyhedral corners, however, $\gamma$ always corresponds to the union of great circles, for which $k=0$, which explains the absence of a $\log ^{2} \delta$ term in eq. (1.6). Instead, $v_{n}\left(\theta_{1}, \theta_{2}, \cdots, \theta_{j}\right)$ can be seen to arise from the constant contribution $b_{0}$ in eq. (1.2) which would require a complicated calculation of an spectral function on $\mathbb{S}^{3}$ with a cut, similarly to what happened for the corner function $a_{n}^{(3)}(\Omega)$. An explicit calculation of $v(\pi / 2, \pi / 2, \pi / 2)$ for a trihedral corner in the so-called "Extensive Mutual Information Model" is also provided.

$\diamond$ In section 3, we consider the entanglement entropy of wedge regions in $d=4$. First, we compute the wedge function $f(\Omega)$, as defined in eq. (1.7), in the nearly smooth 
limit for general CFTs. Then, we argue that the entanglement entropy of a massive free field in $d=3$ corresponding to a corner region can be related to the one corresponding to the wedge in $d=4$. Using this, we show that the angular dependence of $a^{(3)}(\Omega)$ and $f(\Omega)$ do agree with each other, whereas the overall normalization of $f(\Omega)$ is nonuniversal due to the presence of different IR and UV regulators along the transverse and longitudinal directions. Then, we revisit the holographic results for both entangling regions and show that, contrary to the claim in [57], the angular dependence of both functions is in fact different in that case, and therefore for general CFTs.

$\diamond$ In section 4, we consider entangling regions with sharpened and smoothened corners. First, we show that, contrary to common belief, the mutual information of two regions which touch at a point is not necessarily divergent as long as the contact occurs through a sufficiently sharp corner. Then, we argue that the Rényi entropy of regions containing geometric singularities does not always modify the structure eq. (1.2) characteristic of smooth entangling regions. On the other hand, when the corners are sharper than in the usual straight corner case, the usual logarithmic divergence gets replaced by a more divergent term which, nonetheless, never surpasses the area law one.

$\diamond$ In appendix A, we perform an explicit calculation of the cone function $a^{(4)}(\Omega)$ in the Extensive Mutual Information model and show that it agrees with the result valid for general CFTs.

$\diamond$ In appendix B, we use the explicit formula obtained in section 2.3 for the Rényi entropy of elliptic cones, to show that circular cones locally maximize the Rényi entropy within the class of (fixed-area) elliptic cones.

$\diamond$ Finally, in appendix $\mathrm{C}$ we show that the dependence on the opening angle of the (hyper)cone function $a_{n}^{(d)}(\Omega)$ for arbitrary even-dimensional CFTs is given by the four-dimensional result, $\cos ^{2} \Omega / \sin \Omega$ times a linear combination of the form: $\gamma_{0, n}^{(d)}+$ $\gamma_{1, n}^{(d)} \cos (2 \Omega)+\gamma_{2, n}^{(d)} \cos (4 \Omega)+\cdots+\gamma_{(d-4) / 2, n}^{(d)} \cos ((d-4) \Omega)$ for certain theory-dependent quantities $\gamma_{i, n}^{(d)}$ related to the trace-anomaly charges for $n=1$.

\section{Vertex-induced universal terms}

In this section we study the universal contributions to the Rényi entropy arising when the entangling region contains vertices. In the case of a free scalar field, a radial dimensional reduction and a mapping of the problem to $(d-1)$-dimensional de Sitter space allows us to identify the appearance of a quadratically-logarithmic term of the form eq. (1.8) in $d=4$. We show that this term accounts for the result obtained using Solodukhin's formula for (elliptic) cones. In the case of polyhedral corners, our result allows for a

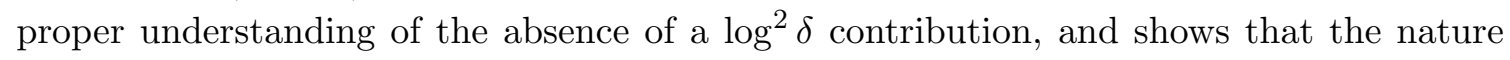
of the remaining logarithmic coefficient - $v_{n}\left(\theta_{1}, \theta_{2}, \cdots, \theta_{j}\right)$ in eq. (1.6) - is intrinsically non-local. 


\subsection{Dimensional reduction for free fields}

Let us consider a real 1-component scalar field of mass $m$ in $d$ spacetime dimensions. The Rényi and entanglement entropies of a subregion $V$ in the groundstate can be obtained from $[17]$

$$
\begin{gathered}
S_{n}(V)=\frac{1}{1-n} \log \left(\operatorname{tr} \rho_{V}^{n}\right)=\frac{1}{1-n} \sum_{k=0}^{n-1} \log Z\left[e^{2 \pi i \frac{k}{n}}\right], \\
S_{\mathrm{EE}}(V) \equiv \lim _{n \rightarrow 1} S_{n}(V)=-\int_{0}^{\infty} d t \frac{\pi}{\cosh ^{2}(\pi t)} \log Z\left[-e^{2 \pi t}\right],
\end{gathered}
$$

where $Z\left[e^{2 \pi i a}\right]$ is the partition function on $\mathbb{R}^{d}$ corresponding to a field which picks up a phase $e^{2 \pi i a}$ when the entangling region $V$ is crossed. The expression for $S_{n}$ follows from a diagonalization procedure in replica space [17].

The partition function $Z\left[e^{2 \pi i a}\right]$ can be computed by exploiting the relation between the free energy and the trace of the Green function of the associated Laplacian operator

$$
\partial_{m^{2}} \log Z\left[e^{2 \pi i a}\right]=-\frac{1}{2} \int_{\mathbb{R}^{d}} d^{d} \vec{r} G_{a}(\vec{r}, \vec{r}),
$$

where

$$
\begin{aligned}
\left(-\nabla_{\vec{r}_{1}}^{2}+m^{2}\right) G_{a}\left(\vec{r}_{1}, \vec{r}_{2}\right) & =\delta\left(\vec{r}_{1}-\vec{r}_{2}\right), \\
\lim _{\epsilon \rightarrow 0^{+}} G_{a}\left(\vec{r}_{1}+\epsilon \vec{\eta}, \vec{r}_{2}\right) & =e^{2 \pi i a} \lim _{\epsilon \rightarrow 0^{+}} G_{a}\left(\vec{r}_{1}-\epsilon \vec{\eta}, \vec{r}_{2}\right), \quad \vec{r}_{1} \in V,
\end{aligned}
$$

where $\vec{\eta}$ is orthogonal to $V$. On general grounds, the Green function can be written in terms of the eigenfunctions and eigenvalues of the Laplacian as

$$
G_{a}\left(\vec{r}_{1}, \vec{r}_{2}\right)=\int d \lambda \frac{\psi_{\lambda}\left(\vec{r}_{1}\right) \psi_{\lambda}^{*}\left(\vec{r}_{2}\right)}{\lambda^{2}+m^{2}}, \quad \text { where } \quad \nabla_{\vec{r}_{1}}^{2} \psi_{\lambda}\left(\vec{r}_{1}\right)=-\lambda^{2} \psi_{\lambda}\left(\vec{r}_{1}\right)
$$

In situations in which the boundary conditions eq. (2.5) are implemented along the angular directions, it is possible to perform a separation of variables between the radial and angular components of $\psi_{\lambda}(x)$. This will be the case for cones of different sections or polyhedral corners. We can write

$$
\psi_{\lambda}(\vec{r})=\sum_{\ell} F_{\ell, \lambda}(r) \Phi_{\ell}\left(\Omega_{\mathbb{S}^{d-1}}\right), \quad \text { with } \quad \nabla_{\mathbb{S}^{d-1}}^{2} \Phi_{\ell}\left(\Omega_{\mathbb{S}^{d-1}}\right)=-\ell(\ell+d-2) \Phi_{\ell}\left(\Omega_{\mathbb{S}^{d-1}}\right),
$$

where the eigenfunctions of $\nabla_{\mathbb{S}^{d-1}}^{2}$ will satisfy different boundary conditions inherited from eq. (2.5) depending on the entangling region. Those boundary conditions will also determine the possible values of $\ell$ appearing in eq. (2.7), which will not correspond to integer numbers in general - hence, the parametrization of the Laplacian eigenvalues used in eq. (2.7) is just a convenient choice. In spherical coordinates, the $d$-dimensional Laplace operator in eq. (2.6) becomes

$$
\nabla_{\vec{r}}^{2}=\frac{\partial^{2}}{\partial r^{2}}+\frac{(d-1)}{r} \frac{\partial}{\partial r}+\frac{1}{r^{2}} \nabla_{\mathbb{S}^{d-1}}^{2}
$$


Then, it is straightforward to find the equation for the radial component of $\psi_{\lambda}(\vec{r})$, namely

$$
\ddot{F}_{\ell, \lambda}(r)+\frac{(d-1)}{r} \dot{F}_{\ell, \lambda}(r)-\frac{\left[\ell(\ell+d-2)-\lambda^{2} r^{2}\right]}{r^{2}} F_{\ell, \lambda}(r)=0 .
$$

The general solution to this equation is given by

$$
F_{\ell, \lambda}(r)=r^{-\frac{(d-2)}{2}}\left[\alpha_{\ell, \lambda} J_{\ell+\frac{d-2}{2}}[\lambda r]+\beta_{\ell, \lambda} Y_{\ell+\frac{d-2}{2}}[\lambda r]\right],
$$

where $J_{s}[x]$ and $Y_{s}[x]$ are Bessel functions of the first and second kind, respectively, and $\alpha_{\ell, \lambda}$ and $\beta_{\ell, \lambda}$ are integration constants. $Y_{s}[x]$ blows up at $x=0$, so we set $\beta_{\ell, \lambda}=0 .{ }^{5}$ On the other hand, the orthogonality relation ${ }^{6}$

$$
\int_{0}^{\infty} r^{d-1} d r \int d \Omega \psi_{\ell, \lambda}(\vec{r}) \psi_{\ell^{\prime}, \lambda^{\prime}}^{*}(\vec{r})=\delta_{\ell \ell^{\prime}} \delta\left(\lambda-\lambda^{\prime}\right)
$$

fixes the remaining integration constant to $\alpha_{\ell, \lambda}=\sqrt{\lambda}$. Using this information, we are ready to rewrite the Green function in eq. (2.6) as

$$
G_{a}\left(\vec{r}_{1}, \vec{r}_{2}\right)=\sum_{\ell, \ell^{\prime}} r_{1}^{-\frac{(d-2)}{2}} r_{2}^{-\frac{(d-2)}{2}} \Phi_{\ell}\left(\Omega_{1}\right) \Phi_{\ell^{\prime}}^{*}\left(\Omega_{2}\right) \int d \lambda \frac{\lambda}{\lambda^{2}+m^{2}} J_{\ell+\frac{d-2}{2}}\left[\lambda r_{1}\right] J_{\ell^{\prime}+\frac{d-2}{2}}\left[\lambda r_{2}\right]
$$

We can now write the trace of the Green function appearing in eq. (2.3) as

$$
\begin{aligned}
-\int_{\mathbb{R}^{d}} d^{d} \vec{r} G_{a}(\vec{r}, \vec{r}) & =-\sum_{\ell} \int_{0}^{\infty} d r r I_{\ell+\frac{d-2}{2}}[m r] K_{\ell+\frac{d-2}{2}}[m r] \\
& =-\sum_{\ell}\left(\int_{0}^{\infty} \frac{d r}{2 m}\right)+\frac{1}{2 m^{2}} \sum_{\ell}\left|\ell+\frac{d-2}{2}\right|,
\end{aligned}
$$

where in the first line we performed the integration over the angles, and used the following result to evaluate the $\lambda$ integral:

$$
\int_{0}^{\infty} d \lambda \frac{\lambda}{\lambda^{2}+m^{2}} J_{\ell+\frac{d-2}{2}}[\lambda r]^{2}=I_{\ell+\frac{d-2}{2}}[m r] K_{\ell+\frac{d-2}{2}}[m r],
$$

where $I_{s}[x]$ and $K_{s}[x]$ are the modified Bessel functions of the first and second kinds, respectively. To get eq. (2.15), we used the fact that $I_{s}[m r] K_{s}[m r] \rightarrow 1 /(2 m r)$ at large $m r$.

As we can see in eq. (2.15), we obtain two terms. The first is manifestly divergent, and will be responsible for non-universal terms, such as the area law. We ignore this nonuniversal contribution from now on - which, besides, has no dependence on the angles and focus on the second. When integrated over $m^{2}$ to obtain $\log Z\left[e^{2 \pi i a}\right]$, this term will

\footnotetext{
${ }^{5}$ Observe that $Y_{\alpha}[x]$ can be written as a linear combination of $J_{\alpha}[x]$ and $J_{-\alpha}[x]: Y_{\alpha}[x]=J_{\alpha}[x] \cot (\alpha \pi)-$ $J_{-\alpha}[x] / \sin (\alpha \pi)$. Setting $\beta_{\ell, \lambda}=0$ implicitly selects the sign of $\alpha$ in $J_{\alpha}[x]$ to be positive: negative values of $\alpha$ are the ones responsible for the blow up of $Y_{\alpha}[x]$ at $x=0$.

${ }^{6}$ Here, we use the orthogonality relations

$$
\sqrt{\lambda \lambda^{\prime}} \int_{0}^{\infty} d r r J_{s}[r \lambda] J_{s}\left[r \lambda^{\prime}\right]=\delta\left(\lambda-\lambda^{\prime}\right), \quad \text { and } \quad \int d \Omega \Phi_{\ell}(\Omega) \Phi_{\ell^{\prime}}^{*}(\Omega)=\delta_{\ell \ell^{\prime}}
$$


yield a logarithmically divergent contribution to the Rényi and entanglement entropies. Completing squares, we can finally write

$$
\partial_{m^{2}} \log Z\left[e^{2 \pi i a}\right]=\frac{1}{4 m^{2}} \operatorname{tr} \sqrt{-\nabla_{\mathbb{S}^{d-1}}^{2}+\frac{(d-2)^{2}}{4}},
$$

which can be alternatively written as

$$
\partial_{m^{2}} \log Z\left[e^{2 \pi i a}\right]=\frac{1}{4 \pi m^{2}} \int_{0}^{\infty}\left[\xi^{1 / 2} \operatorname{tr}\left[\frac{1}{\nabla_{\mathbb{S}^{d-1}}^{2}-\frac{(d-2)^{2}}{4}-\xi}\right]+\xi^{-1 / 2}\right] d \xi
$$

The term involving integration over $\xi^{-1 / 2}$ produces another angle-independent nonuniversal term which we also ignore. Integrating over $m^{2}$, it follows that

$$
\left.\log Z\left[e^{2 \pi i a}\right]\right|_{\log }=-\frac{\log (\delta / L)}{2 \pi} \int_{0}^{\infty} \xi^{1 / 2} \operatorname{tr}\left[\frac{1}{\nabla_{\mathbb{S} d-1}^{2}-\frac{(d-2)^{2}}{4}-\xi}\right] d \xi,
$$

where " $\mid \log "$ makes explicit the fact that we are omitting additional contributions coming from the first term in eq. (2.15) as well as the last term in eq. (2.18). Here, $L$ is a longdistance quantity parametrizing the linear size of subregion $V$.

In the prototypical case of a corner region of opening angle $\Omega$ in $d=3$, the problem gets reduced to computing the trace of the Green function on a sphere $\mathbb{S}^{2}$ with a cut of angle $\Omega$. The corresponding boundary conditions resulting from eq. (2.5) read in that case

$$
\lim _{\epsilon \rightarrow 0^{+}} \Phi_{\ell}(\pi / 2+\epsilon, \phi)=e^{2 \pi i a} \lim _{\epsilon \rightarrow 0^{+}} \Phi_{\ell}(\pi / 2-\epsilon, \phi), \quad \phi \in[-\Omega / 2, \Omega / 2] .
$$

The cut is therefore an angular sector on the equatorial $\mathbb{S}^{1}$. If we consider entangling regions emanating from a vertex in $d=4$, the corresponding cut on $\mathbb{S}^{3}$ will correspond to some area on the surface of a $\mathbb{S}^{2}$. The boundary of the intersection region, which in the case of the $d=3$ corner is just the union of two points, corresponds in this case to some curve $\gamma$ on the surface of the $\mathbb{S}^{2}$ - see figure 1 for the case of a cone and a trihedral corner.

Interestingly, the trace of the Green function which appears inside the integral can be related to a $(d-1)$-dimensional Rényi entropy in de Sitter (dS) space [15]. In particular, if one considers the Rényi entropy for some region in $\mathrm{dS}_{(d-1)}$ for a scalar field coupled to the background curvature through a term $g(d) R \phi^{2}$, where $R$ is the scalar curvature and $g(d)$ some function of the dimension, ${ }^{7}$ the trace of the Green function appearing in eq. (2.3) can be read straightforwardly, and it follows that

$$
\partial_{m^{2}} \log Z\left[\mathrm{dS}_{(d-1)}\right]=\frac{1}{2} \operatorname{tr}\left[\frac{1}{\nabla_{\mathbb{S}^{d-1}}^{2}-d(d-1) g(d)-m^{2}}\right],
$$

where we used $R=d(d-1) / L_{\mathrm{dS}}^{2}$, and we set the $\mathrm{dS}$ radius to unity from now on, $L_{\mathrm{dS}}=$ 1. Observe that eq. (2.21) is very similar to the quantity appearing in the integrand of

\footnotetext{
${ }^{7}$ Note that if $g(d)=(d-2) /(4(d-1))$, the scalar is conformally coupled.
} 

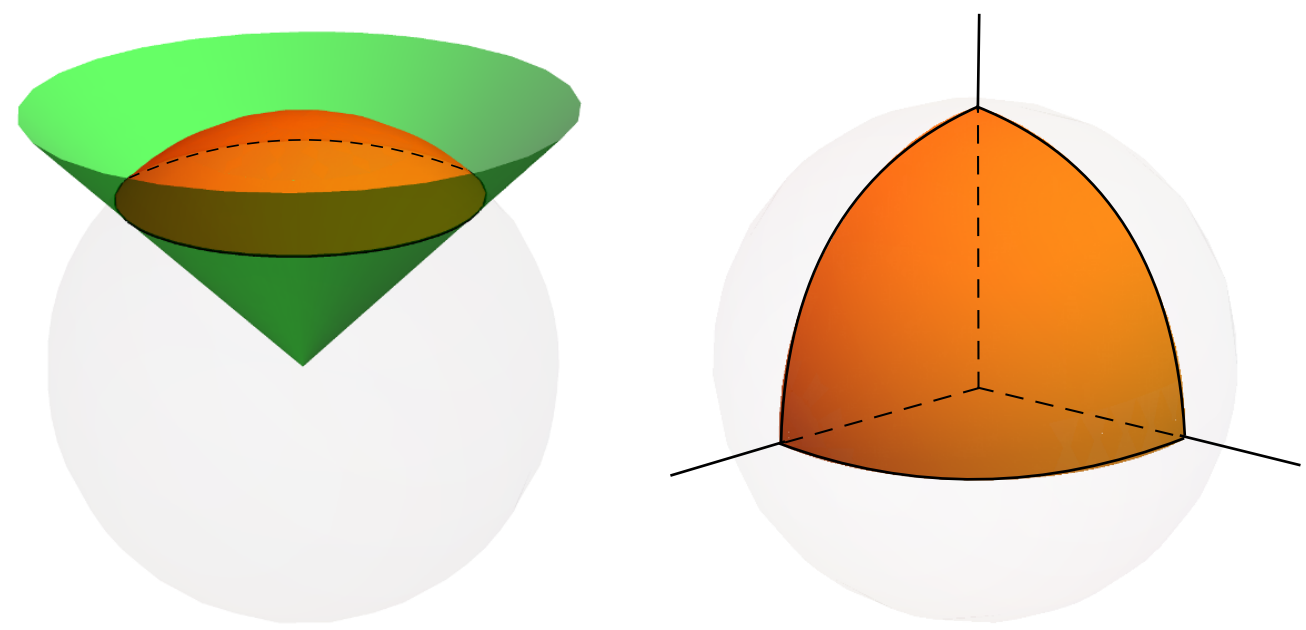

Figure 1. (Left) We plot the $\mathbb{S}^{1}$ resulting from the intersection of the conical region defined by $\theta \in[-\Omega, \Omega], \phi \in[0,2 \pi)$ with a unit $\mathbb{S}^{2}$ in the equator of $\mathbb{S}^{3}$. The boundary conditions are implemented as we approach the orange region in the equator of $\mathbb{S}^{3}$ from above and below in the additional angular coordinate (not shown in the figure). (Right) We plot the intersection of a trihedral corner region of right opening angles with the unit $\mathbb{S}^{2}$. In this case, $\gamma$ is the union of three quarters of great circles, for which the extrinsic curvature vanishes, $k=0$.

eq. (2.19), as long as the boundary conditions imposed in both cases match. Then, if we choose $g(d)=(d-2)^{2} /(4 d(d-1))$, we can use eq. (2.1) to write ${ }^{8}$

$$
\left.S_{n}\right|_{\log }=-\frac{\log (\delta / L)}{\pi} \int_{0}^{\infty} d m^{2} m \frac{\partial S_{n}^{\mathrm{dS}_{(d-1)}}}{\partial m^{2}},
$$

where in the l.h.s. we have the Rényi entropy corresponding to the region $V$ in $\mathbb{R}^{d}$, and in the r.h.s., $S_{n}^{\mathrm{dS}_{(d-1)}}$ stands for the Rényi entropy in $\mathrm{dS}_{(d-1)}$ corresponding to a region with the same boundary conditions as those inherited for $V$ from eq. (2.5) on the angular coordinates. We can extract some useful information from $S_{n}^{\mathrm{dS}_{(d-1)}}$. The corresponding Rényi entropy admits a high-mass expansion of the form

$$
S_{n}^{\mathrm{dS}_{(d-1)}}=c_{n,(d-3)} m^{d-3}+\cdots+c_{n, 0}+\frac{c_{n,-1}}{m}+\cdots
$$

valid for masses much greater than the de Sitter inverse radius, $m \gg 1$. Recall that in our units $L_{\mathrm{dS}}=1$. Observe that in this expansion, we have hidden terms involving various powers of the corresponding UV cutoff $\delta$ inside the coefficients $c_{n, q}$ - which have dimensions of (length) $)^{q}$ for each $q$. In principle, one could naively think of various dimensionless combinations of $m, \delta$ and local integrals over the entangling surface susceptible of appearing in eq. (2.23). However, divergent contributions involving $\delta$ originate from the (massless) UV theory and they are controlled by local integrals over the entangling surface. The effects

\footnotetext{
${ }^{8}$ If one chooses the conformally coupled value of $g(d)$, the result doesn't change much. The only difference is that the derivative is to be evaluated at some other $m,\left.\int_{0}^{\infty} d m^{\prime 2} m^{\prime}\left[\frac{\partial S_{n}^{\mathrm{dS}}(d-1)}{\partial m^{2}}\right]\right|_{m=m^{\prime}+f(d)}$. The choice doesn't affect any of the conclusions that follow.
} 
of introducing a mass translate into corrections to such contributions which disappear as $m \rightarrow 0$. This forbids the presence of non-vanishing terms involving negative powers of $m$ combined with $\delta$. Terms with negative powers of $m$ not involving $\delta$ can however appear weighted by the appropriate local integrals. ${ }^{9}$ This is the case of $\mathcal{O}\left(\mathrm{m}^{-1}\right)$ term in eq. (2.23). The coefficient $c_{n,-1}$ has dimensions of (length) ${ }^{-1}$ and, as we say, it must be given by an integral over the boundary of the entangling region. In $d=4$, the only term satisfying these properties we can write reads

$$
c_{n,-1}=\alpha_{n} \int_{\gamma} k^{2}
$$

where $\gamma$ is indeed the boundary of the entangling region in $\mathrm{dS}_{3}$ (which corresponds to the intersection of the original region $V$ with the unit $\mathbb{S}^{2}$ mentioned above), $k$ is the trace of the extrinsic curvature of $\gamma$, and $\alpha_{n}$ is a dimensionless constant. It is not difficult to see that an analogous term could be written in general even dimensions as $c_{n,-1} \sim \sum_{i} \alpha_{n}^{i} \int_{\Sigma} k_{i}^{d-2}$, where $k_{i}^{d-2}$ would be various independent contractions of extrinsic curvatures of order $(d-2)$ - or intrinsic curvatures of order $(d-2) / 2$. In odd dimensions, however, we are forced to set $c_{n,-1}=0$. This is because any possible contraction of an odd number of extrinsic curvatures produces a term which would change sign if instead of considering $V$ we chose its complement as our entangling region. However, the purity of the groundstate forbids this - for related discussions see e.g., [3, 4]. In the rest of the section we will thus assume that $d$ is even. Observe also that, in principle, terms involving the curvature of the background manifold could have appeared as well. However, those would be independent of the geometry of $\gamma$. Then, since we know that for a "cone" of opening angle $\Omega=\pi$ - for which $\gamma$ is just the equator of the $\mathbb{S}^{2}$ — there is no $\log ^{2} \delta$ contribution at all, the corresponding coefficients must be zero.

As we have said, the local nature of $c_{n,-1}$ prevents it from feeling the curvature of the background geometry. Hence, the coefficient $\alpha_{n}$ can be for example connected to the result corresponding to a cylinder entangling region in flat space, which fixes $\alpha_{n}=\frac{1}{8} f_{b}(n)$, where $f_{b}(n)$ is one of the coefficients appearing in the universal contribution for smooth entangling surfaces $[7,8]$ - see eq. (2.42) below. Indeed, in the case of the cylinder, a longitudinal dimensional reduction can be performed, effectively reducing the problem to that of a massive field in Minkowski 3 . A mass expansion analogous to eq. (2.23) holds in that case, and the term yielding the usual universal (logarithmic) contribution also comes from the $1 / \mathrm{m}$ piece. This is controlled by an integral which takes the same form as eq. (2.24). The local nature of that term makes it independent of the global curvature of the spacetime in which the curve is embedded, so the overall coefficient is the same. ${ }^{10}$ Alternatively, we could leave $\alpha_{n}$ undetermined for now, and then fix it by imposing that the final contribution appearing in eq. (2.28) yields the correct answer in a particular case for which the full dependence on the Rényi index is known for general theories - e.g., for a circular straight cone. Naturally, this fixes $\alpha_{n}$ in exactly the same way as using the cylinder result.

\footnotetext{
${ }^{9}$ For discussions on the interplay between mass and UV-divergent terms see e.g., [63, 64].

${ }^{10}$ For a free scalar and a free Dirac field, this cylinder dimensional reduction was explicitly carried out in [65], yielding excellent agreement with the general-CFT expectation.
} 
All in all, we are left with a contribution of the form

$$
\left.S_{n}^{\left(\mathrm{dS}_{3}\right)}\right|_{m^{-1}}=\frac{f_{b}(n)}{8 m} \int_{\gamma} k^{2} .
$$

Inserting this in the integral appearing in eq. (2.22), we find

$$
\begin{aligned}
\int_{1 / L^{2}}^{1 / \delta^{2}} d m^{2} m \frac{\left.\partial S_{n}^{\left(\mathrm{dS}_{3}\right)}\right|_{m^{-1}}}{\partial m^{2}} & =-\frac{1}{8} \int_{1 / L^{2}}^{1 / \delta^{2}} d m^{2} m \frac{f_{b}(n)}{2 m^{3}} \int_{\gamma} k^{2} \\
& =\frac{1}{16} f_{b}(n)\left[\log \left(\delta^{2} / L^{2}\right)+\mathcal{O}(1)\right] \int_{\gamma} k^{2}
\end{aligned}
$$

where we introduced a regulator for high masses. As we can see, this produces an additional logarithmic divergence which, when combined with the one already present in eq. (2.22), gives rise to the term

$$
\left.S_{n}\right|_{\log ^{2}}=\frac{-f_{b}(n)}{8 \pi} \log ^{2} \delta \int_{\gamma} k^{2} .
$$

Crucially, a contribution like eq. (2.28) will only be present for entangling regions such that the extrinsic curvature of $\gamma$ is nonvanishing. This will be the case of straight cones with various cross-sections, but not of polyhedral corners, as we explain now.

\subsection{Polyhedral corners}

In the case of entangling regions involving polyhedral corners, the intersection of $\partial V$ with the unit $\mathbb{S}^{2}$ will be the union of portions of great circles - see figure 1 - for which $k=0$. This explains why, as previously observed [58-62], trihedral corners have a universal $\log \delta$ divergence, instead of a $\log ^{2} \delta$ one. The above analysis also reveals that, in the case of polyhedral corners, the corresponding logarithmic contribution will not come from some simple local integral along any curve on $\mathbb{S}^{2}$. Rather, its calculation would require the full answer for the spectral function on the sphere with a cut appearing in eq. (2.19) - this is completely analogous to what happens for the usual corner universal term in $d=3$ [1517]. Hence, the function $v_{n}\left(\theta_{1}, \theta_{2}, \cdots, \theta_{j}\right)$ appearing in the logarithmic contribution to the Rényi entropy for polyhedral corners

$$
\left.S_{n}^{\text {polyhedral }}\right|_{\log }=v_{n}\left(\theta_{1}, \theta_{2}, \cdots, \theta_{j}\right) \log (L / \delta),
$$

will generally be a highly non-local term, and there is no reason to expect it to be controlled by a linear combination of trace-anomaly coefficients (or their Rényi entropy generalizations, $f_{a}(n)$ and $\left.f_{b}(n)\right)$ as suggested in [60,61]. This observation, while relying on a free-field calculation, should be valid for general CFTs.

On the other hand, one does expect that in the limit of a very open polyhedral corner ("almost smooth"), $v_{1}\left(\theta_{1}, \theta_{2}, \cdots, \theta_{j}\right)$ is nevertheless controlled by the stress-tensor twopoint function charge [51] $C_{T} \propto c$, in line with the results of [28, 46, 47, 52, 53]. For general CFTs, it was indeed shown that trihedral corners in $d=4$ receive a contribution $\propto c \log (L / \delta)$ in the almost smooth limith [62]. In the following subsection, we give an analytical calculation of the polyhedral contribution away from the flat limit using a special model. 


\subsubsection{Cubes in the extensive mutual information model}

For entangling regions containing vertices that are not near the flat limit, one is left with very few analytical methods. One particularly useful tool is the so-called "Extensive Mutual Information Model" (EMI) [66-68]. The EMI is not defined through a Lagrangian, but instead allows for a simple geometric computation of the entanglement entropy consistent with conformal symmetry, and has passed several non-trivial tests in all dimensions [18, $46,66,68]$. As its name suggests, the characterizing feature of this model is the fact that the mutual information satisfies the extensivity property

$$
I(A, B)+I(A, C)=I(A, B \cup C) .
$$

This requirement strongly constrains the form of the entanglement entropy and the mutual information. In particular, the entanglement entropy of a region $A$ in the EMI is defined through the following integral: ${ }^{11}$

$$
S^{\mathrm{EMI}}=\kappa \int_{\partial A} d^{d-2} \mathbf{r}_{1} \int_{\partial A} d^{d-2} \mathbf{r}_{2} \frac{\mathbf{n}_{1} \cdot \mathbf{n}_{2}}{\left|\mathbf{r}_{1}-\mathbf{r}_{2}\right|^{2(d-2)}},
$$

where $\mathbf{n}$ is the normal vector to the boundary of $A, \partial A . \kappa$ is a positive parameter. In this section, we drop the subscript "EE", as the EMI ansatz can be trivially adapted to general Rényi entropies $S_{n}$ by replacing $\kappa$ by $\kappa_{n}$.

Cubic trihedral. We now analytically calcualte the full entanglement entropy for the cubic trihedral, see figure 1 , with $\theta_{1}=\theta_{2}=\theta_{3}=\pi / 2$, using the EMI. In other words, region $A$ corresponds to an octant of $\mathbb{R}^{3}$, say $x, y, z>0$. Since any pair of faces has mutually orthogonal normals, we are left with contributions where both $\mathbf{r}_{1}$ and $\mathbf{r}_{2}$ lie on the same face, which we take to be in the $x y$-plane:

$$
S=3 \kappa \int_{0}^{\infty} d x_{1} d x_{2} d y_{1} d y_{2} \frac{1}{\left[\left(x_{1}-x_{2}\right)^{2}+\left(y_{1}-y_{2}\right)^{2}\right]^{2}} .
$$

We first perform the $x_{1}$ integral, then the $x_{2}$ one, for which a finite long-distance cutoff $L$ is needed to avoid the divergence:

$$
S=3 \kappa \int_{0}^{\infty} d y_{1} d y_{2} L \frac{2 \tan ^{-1}\left(\frac{L}{y_{1}-y_{2}}\right)+\pi \operatorname{sgn}\left(y_{1}-y_{2}\right)}{4\left(y_{1}-y_{2}\right)^{3}} .
$$

To perform the integral over $y_{1}$, we break it up into two parts to avoid the short distance divergence occuring when $y_{1} \rightarrow y_{2}: \int_{0}^{\infty} d y_{1}=\int_{0}^{y_{2}-\delta} d y_{1}+\int_{y_{2}+\delta}^{\infty} d y_{1}$. The result is

$$
\begin{aligned}
I=\int_{\delta}^{L} d y_{2} \frac{1}{4 L}\left[\frac{L(L \pi-2 \delta)}{\delta^{2}}\right. & +2\left(\frac{L^{2}}{\delta^{2}}+1\right) \cot ^{-1}(\delta / L)+\frac{L}{y_{2}} \\
& \left.-\frac{L^{2} \pi}{2 y_{2}^{2}}-\left(\frac{L^{2}}{y_{2}^{2}}+1\right) \cot ^{-1}\left(y_{2} / L\right)\right] .
\end{aligned}
$$

\footnotetext{
${ }^{11}$ A somewhat similar formula for the entanglement entropy of a free Fermi gas was obtained in [69].
} 
This last integral needs to be performed with both short and long distance cutoffs, which can be done exactly. The final result for $S$, expanded at $\delta / L \ll 1$, reads

$$
S=3 \kappa I=3 \kappa\left(\frac{\pi L^{2}}{2 \delta^{2}}-\left(1+\frac{3 \pi}{4}\right) \frac{L}{\delta}+\frac{1}{2} \ln (L / \delta)+\cdots\right) .
$$

This contains the area law, a negative contribution from the edges $\propto L / \delta$, and the positive trihedral contribution governed by

$$
v(\pi / 2, \pi / 2, \pi / 2)=\frac{3}{2} \kappa
$$

which is positive since $\kappa>0$.

Finite cube. For a finite cube of linear size $L$ we have 2 types of contributions: $\mathbf{r}_{1}$ and $\mathbf{r}_{2}$ lying on the same face or on opposite faces. The latter contribution can be omitted for the purpose of determining the singular contributions (dependent on $\delta$ ) because $\left|\mathbf{r}_{1}-\mathbf{r}_{2}\right| \geq L$, which implies that the corresponding integral for $S$ contains no short-distance divergences. It is in fact a pure number, as can be verified by an explicit calculation.

We thus need to evaluate an integral analogous to the above one but with finite support on a face of the cube:

$$
S=6 s_{1} I=6 s_{1} \int_{0}^{L} d x_{1} d x_{2} d y_{1} d y_{2} \frac{1}{\left[\left(x_{1}-x_{2}\right)^{2}+\left(y_{1}-y_{2}\right)^{2}\right]^{2}} .
$$

The factor of 6 counts the number of faces of the cube. After performing the integrals over $x_{1}, x_{2}$, we get

$$
I=\int_{0}^{L} d y_{1} d y_{2} \frac{L}{\left(y_{1}-y_{2}\right)^{3}} \tan ^{-1}\left(\frac{L}{y_{1}-y_{2}}\right) .
$$

We perform the $y_{1}$ integral as above by splitting it to avoid the $y_{1} \rightarrow y_{2}$ divergence. The result reads

$$
\begin{aligned}
I=\int_{\bar{\delta}}^{1-\bar{\delta}} & \frac{2\left(\bar{\delta}^{2}+1\right)}{2 \bar{\delta}^{2}\left(\bar{y}_{2}-1\right)^{2} \bar{y}_{2}^{2}}\left\{\left(\bar{y}_{2}-1\right)^{2} \bar{y}_{2}^{2} \cot ^{-1}(\bar{\delta})-\bar{\delta}\left[\bar{\delta}\left(\left(\bar{y}_{2}-2\right) \bar{y}_{2}+2\right) \bar{y}_{2}^{2} \cot ^{-1}\left(1-\bar{y}_{2}\right)\right.\right. \\
& \left.\left.+\left(\bar{y}_{2}-1\right)\left(\bar{y}_{2}\left(\bar{\delta}+2\left(\bar{y}_{2}-1\right) \bar{y}_{2}\right)+\bar{\delta}\left(\bar{y}_{2}-1\right)\left(\bar{y}_{2}^{2}+1\right) \cot ^{-1}\left(\bar{y}_{2}\right)\right)\right]\right\} d \bar{y}_{2}, \quad(2.39)
\end{aligned}
$$

where the bar variables are normalized by $L$. In contrast with the semi-infinite trihedral calculation, we note that the final integral has divergences at both $y_{2}=0, L$, which is a consequence of the finiteness of the cube. Performing the integral and Taylor expanding the answer in powers of $L / \delta$, we obtain

$$
S=6 \kappa I=6 \kappa\left(\frac{\pi L^{2}}{2 \delta^{2}}-\left(2+\frac{3 \pi}{2}\right) \frac{L}{\delta}+2 \ln (L / \delta)+\cdots\right) .
$$

As expected, we thus find that the logarithmic coefficient of the cube is 8 times that of a single $\pi / 2$ trihedral corner:

$$
(\log \text { coefficient for cube })=8 v(\pi / 2, \pi / 2, \pi / 2)=12 \kappa .
$$

As a further check, the divergence due to the 12 edges of the cube, $-6 \kappa\left(2+\frac{3 \pi}{2}\right) L / \delta$, is 4 times that of the one found for a single cubic trihedral which has 3 edges, see eq. (2.35). 
We emphasize that for polyhedral corners, even though terms of the form $L / \delta$ due to the presence of edges appear, the contribution from the corner itself is logarithmic, just like for smooth surfaces - see eq. (2.42) below. We are used to see that the presence of singularities on the entangling surface modifies the structure of divergences in the Rényi entropy, so this match on the type of universal divergence may look surprising at first. In section 4 we will provide genuine examples of entangling regions which do not modify the divergences structure. In the present case, however, the coincidence is accidental, as should be clear from the discussion above: while the universal logarithmic term is controlled by a local integral on the entangling surface for smooth regions, we expect the one corresponding to a polyhedral corner to involve a complicated theory-dependent function. In a setup in which we got to the polyhedral corner from a limiting procedure on a smooth region, the logarithm would not emerge from the already present logarithmic term produced locally near the edges (which would be enhanced to a $\log ^{2}$ term and would vanish for the reasons already explained), but rather from the constant one. In this regard, note that the presence of a new regulator, $\epsilon$, which would smooth out the wedges and trihedral corners would give rise to logarithmic contributions of the form $\log (\epsilon / \delta)$, which in the $\epsilon \rightarrow 0$ limit would contaminate the coefficient of the $\log L$ term, preventing it from being controlled by $f_{b}(n)$ as one would have naively guessed from the coefficient of the $\log \delta$ term in Solodukhin's formula eq. (2.42).

\subsection{Elliptic cones}

Let us now turn to the case of (elliptic) conical entangling surfaces, as shown in figure 2. For those, the trace of the extrinsic curvature is non-zero, $k \neq 0$, and a $\log ^{2} \delta$ universal contribution of the form eq. (2.28) is present. Eq. (2.28) suggests that, on general grounds, the dependence on the geometric properties of the corresponding entangling surface is theory-independent, the only information about the theory in question being encoded in the overall $f_{b}(n)$ coefficient. The result for this universal contribution in the case of cones with circular cross-sections is known to be obtainable for general CFTs [57] from Solodukhin's formula [7] — see eq. (2.42) — up to a missing $1 / 2$ factor whose origin we discuss below. Here, we verify that the free-field expression eq. (2.28) exactly produces the result predicted by Solodukhin's formula both for circular cones, and in the less trivial case of cones with elliptic cross sections, for which we compute the corresponding universal coefficient explicitly in terms of the elliptic cross-sections eccentricity.

\subsubsection{General CFTs}

When the entangling surface $\Sigma$ is smooth, the corresponding universal contribution can be obtained using Solodukhin's formula ${ }^{12}[7,8]$,

$$
S_{n}^{\text {univ }}=\int_{\Sigma} d^{2} y \sqrt{h}\left[f_{a}(n) \mathcal{R}+f_{b}(n)\left(\operatorname{tr} k^{2}-\frac{1}{2} k^{2}\right)-f_{c}(n) C_{a b} a b\right] \frac{\log \delta}{2 \pi} .
$$

\footnotetext{
${ }^{12}$ In this expression, $h_{a b}$ is the induced metric on $\Sigma$ and $\mathcal{R}$ its associated Ricci scalar. Extrinsic curvatures associated to the two normal vectors $n_{\mu}^{(i)},(i=1,2)$, are denoted by $k_{a b}^{(i)}$, where indices $a, b$ run through the tangent directions to $\Sigma$. Also, $\operatorname{tr} k^{2} \equiv k_{a}^{(i) b} k_{b}^{(i) a}$ and $k^{2} \equiv k_{a}^{(i) a} k_{b}^{(i) b}$, where indices are raised with the inverse metric $h^{a b}$. Furthermore, $C_{a b}{ }^{a b}=0$ is the background Weyl curvature projected on the surface. We will consider a flat background, so we set $C_{a b}{ }^{a b}=0$ henceforth.
} 

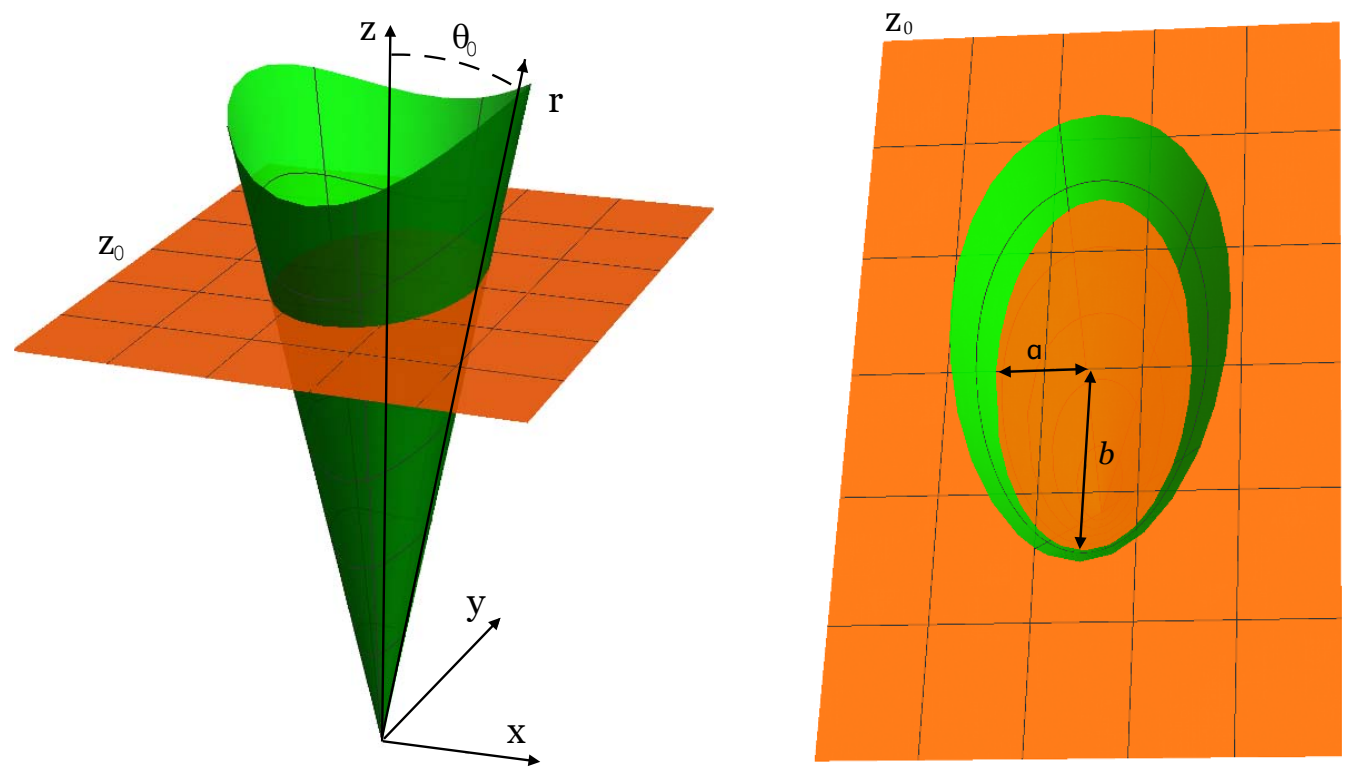

Figure 2. We plot an elliptic cone defined by $\theta=\theta_{0}$ in the coordinates eq. (2.43) (left). The intersection of constant height planes $z=z_{0}$ with the cone form ellipses of semi-minor and semimajor axis $a$ (the direction along which $\theta_{0}$ is defined) and $b$, respectively (right).

The only theory-dependent input appears through functions $f_{a, b, c}(n)$, which do not depend on the geometry of the entangling surface or the background spacetime - in particular, $f_{a}(1)=a, f_{b}(1)=f_{c}(1)=c$, where $a$ and $c$ are the usual trace-anomaly charges. As usual, $\delta$ is a UV regulator, which should appear weighted by some finite dimensionful scale $\ell$ characterizing $\Sigma$. This scale can depend on the various dimensions characterizing the surface but this does not affect the universal coefficient in front of the logarithmic divergence - e.g., if we have two relevant scales, $R_{1}$ and $R_{2}$, the difference between $\log \left(R_{1} / \delta\right)$ and $\log \left(R_{2} / \delta\right)$ is always finite $\sim \log \left(R_{1} / R_{2}\right)$, so it adds up to subleading $\log \delta$ divergences, but does not affect the $\log ^{2} \delta$ one.

Even though eq. (2.42) is in principle not valid when $\Sigma$ includes geometric singularities, it has been argued that it produces the right answer for (circular) conical entangling regions when properly used - see below. We will see that exactly the same treatment applies in the case of elliptic cones, thus allowing us to rely on eq. (2.42) also in that case.

In order to study the Rényi entropy of elliptic cones, we will use sphero-conal coordinates $(r, \theta, \phi)$. These form an orthogonal system of coordinates, and they are defined in terms of the usual Cartesian ones as

$$
x=r \sin \theta \cos \phi, \quad y=r \sqrt{1-\kappa^{2} \cos ^{2} \theta} \sin \phi, \quad z=r \cos \theta \sqrt{1-\left(1-\kappa^{2}\right) \sin ^{2} \phi},
$$

where $r \geq 0,0 \leq \theta \leq \pi, 0 \leq \phi \leq 2 \pi$, and the parameter $\kappa$ is defined such that $0 \leq \kappa \leq 1$. For $\kappa=1$, one recovers the usual spherical coordinates. 
In these coordinates, the metric of four-dimensional Euclidean space reads

$d s^{2}=d t^{2}+d r^{2}+r^{2}\left[\frac{\kappa^{2} \sin ^{2} \theta+\left(1-\kappa^{2}\right) \cos ^{2} \phi}{1-\kappa^{2} \cos ^{2} \theta}\right] d \theta^{2}+r^{2}\left[\frac{\kappa^{2} \sin ^{2} \theta+\left(1-\kappa^{2}\right) \cos ^{2} \phi}{1-\left(1-\kappa^{2}\right) \sin ^{2} \phi}\right] d \phi^{2}$.

Coordinate surfaces $\theta=\theta_{0}$ correspond to semi-infinite elliptic cones with their tip at the origin and their axis along the positive $z$-axis if $\cos \theta_{0} \geq 0$, which we will assume from now on - in particular, we take $0 \leq \theta_{0} \leq \pi / 2$. Cross-sections of the cone with constant- $z$ planes, $z=z_{0}$, are homothetic ellipses described by the equation $x^{2} / a^{2}+y^{2} / b^{2}=1$, where the semi-minor and semi-major axis lengths, $a$ and $b$, read, respectively

$$
a=\frac{z_{0} \sqrt{1-\cos ^{2} \theta_{0}}}{\cos \theta_{0}}, \quad b=\frac{z_{0} \sqrt{1 / \kappa^{2}-\cos ^{2} \theta_{0}}}{\cos \theta_{0}} .
$$

Hence, $\kappa$ can be understood as a parameter controlling how much these ellipses differ from circles. Given $\kappa$, the ellipses are fully characterized by $\theta_{0} \cdot{ }^{13}$ Later on it will be convenient to express $\kappa$ in terms of the ellipses' second eccentricity, defined as

$$
e^{\prime} \equiv \sqrt{b^{2} / a^{2}-1}
$$

The relation reads

$$
\kappa^{2}=\frac{1}{1+e^{\prime 2} \sin ^{2} \theta_{0}} .
$$

Naturally, the circular case, $\kappa=1$, is recovered when $e^{\prime}=0$.

Let us now use Solodukhin's formula to compute the universal contribution to the Rényi entropy for an elliptic conical entangling surface. The induced metric on the cone parametrized by $t=0, \theta=\theta_{0}$ trivially follows from eq. (2.44), and reads

$$
d s_{h}^{2}=d r^{2}+r^{2}\left[\frac{\kappa^{2} \sin ^{2} \theta_{0}+\left(1-\kappa^{2}\right) \cos ^{2} \phi}{1-\left(1-\kappa^{2}\right) \sin ^{2} \phi}\right] d \phi^{2},
$$

where we already made use of eq. (2.48). The Ricci scalar of this metric vanishes, as expected. The normal vectors to the cone read $n^{(1)}=\partial_{t}, n^{(2)}=\frac{1}{\sqrt{g_{\theta \theta}}} \partial_{\theta}$, and the only non-vanishing component of the associated extrinsic curvatures reads

$$
k_{\phi \phi}^{(2)}=\frac{\partial_{\theta} g_{\phi \phi}}{2 \sqrt{g_{\theta \theta}}}=\frac{r \kappa^{2} \sin \theta_{0} \cos \theta_{0} \sqrt{1-\kappa^{2} \cos ^{2} \theta_{0}}}{\sqrt{\kappa^{2} \sin ^{2} \theta_{0}+\left(1-\kappa^{2}\right) \cos ^{2} \phi}\left(1-\left(1-\kappa^{2}\right) \sin ^{2} \phi\right)} .
$$

\footnotetext{
${ }^{13}$ The notion of opening angle is not uniquely defined anymore when $\kappa \neq 1$. Indeed, an elliptic cone has a different opening angle for each value of the angular coordinate $\phi$. We can nevertheless define notions of semi-opening angles, corresponding to the angles along the semi-minor and semi-major axes $a$ and $b$. For the elliptic cones defined by $\theta=\theta_{0}$ in the coordinates above, the semi-opening angle in the $(x, z)$-plane (i.e. in the direction of $a$ ) is precisely given by $\theta_{0}$. The semi-opening angle $\tilde{\theta}_{0}$ in the $(y, z)$-plane (i.e. in the direction of $b$ ) is related to $\theta_{0}$ through$$
\sin ^{2} \tilde{\theta}_{0}=1-\kappa^{2} \cos ^{2} \theta_{0}
$$

Of course, both $\theta_{0}$ and $\tilde{\theta}_{0}$ approach each other as $\kappa \rightarrow 1$, and become equal to the single circular-cone opening angle $\Omega$ in that limit.
} 
This reduces to

$$
k_{\phi \phi}^{(2)}=\frac{r}{2} \sin \left(2 \theta_{0}\right),
$$

for $\kappa=1$, as expected. Using this information, it is easy to find

$$
\operatorname{tr} k^{2}-\frac{1}{2} k^{2}=\frac{1}{2}\left(h^{\phi \phi} k_{\phi \phi}^{(2)}\right)^{2}=\frac{\kappa^{4} \sin ^{2} \theta_{0} \cos ^{2} \theta_{0}\left(1-\kappa^{2} \cos ^{2} \theta_{0}\right)}{2 r^{2}\left(\kappa^{2} \sin ^{2} \theta_{0}+\left(1-\kappa^{2}\right) \cos ^{2} \phi\right)^{3}} .
$$

Now, using eq. (2.42) we find ${ }^{14}$

$$
S_{n}^{\text {univ }}=\frac{f_{b}(n) \cos ^{2} \theta_{0}\left(1+e^{\prime 2}\right)}{\pi \sin \theta_{0}} \int_{0}^{\pi / 2}\left[\frac{\left(1+e^{\prime 2} \sin ^{2} \theta_{0} \cos ^{2} \phi\right)^{-1 / 2} d \phi}{\left(1+e^{\prime 2} \cos ^{2} \phi\right)^{5 / 2}}\right] \int_{\epsilon}^{R} \frac{d r}{r} \log \delta,
$$

where we have expressed the parameter $\kappa$ in terms of $e^{\prime}$ using eq. (2.48). In the above expression we have also introduced an additional cutoff on the cone at $r=\epsilon$. This yields

$$
\int_{\epsilon}^{R} \frac{d r}{r} \log (\delta) \rightarrow-\log (\epsilon) \log (\delta)+\ldots
$$

where the dots denote subleading terms as $\epsilon \rightarrow 0$. In principle, $\epsilon$ can be chosen in different ways, depending on how we regulate the cone at the tip. Combined with $\log \delta$, this yields the quadratically logarithmic divergence characteristic of conical surfaces. In the case there is a single cutoff - the holographic setup would be an example - the two regulators must be related. Naively, one would set $\epsilon=\delta$, but this fails to produce the right result by a $1 / 2$ factor - this mismatch has been mentioned and studied from different perspectives in various papers $[9,18,25,57,70,71]$.

One way to see what is going on involves considering the result for a cylindrical region of (cutoff) length $L$ and radius $\rho$. In that case, the analogous result for the Rényi entropy universal term is given by

$$
S_{n}^{\text {univ }}=-\frac{f_{b}(n)}{2} \frac{L}{\rho} \log \left(\frac{\rho}{\delta}\right) .
$$

If we now consider a thin elliptic cone, the contributions to the Rényi entropy from the infinitesimal cylindrical portions that would build up to produce the cone would be given by

$$
d S_{n}^{\text {univ }}=-\frac{f_{b}(n)}{2} \frac{d r(\rho, \theta, \phi)}{\rho} \log \left(\frac{\rho}{\delta}\right)
$$

where we used the same notation for the spherical radial coordinate $r$ which runs along the cone surface from the tip. As long as the cone is right (i.e. lines of fixed $\theta$ and $\phi$ are straight), the dependence on $\rho$ of $r$ will be such that $r(\rho, \theta, \phi)=\rho g(\theta, \phi)$ for some function of the angular coordinates. Then, one finds

$$
S_{n}^{\text {univ }}=-\frac{f_{b}(n) g(\theta, \phi)}{2} \int_{\delta}^{R} \frac{d r}{r} \log \left(\frac{r}{\delta}\right)+\ldots
$$

\footnotetext{
${ }^{14}$ The analogous contribution proportional to $f_{a}(n)$ vanishes for surfaces formed by straight lines emanating from vertices, such as cones. For those, all the intrinsic curvature is concentrated at the tip of the cone, since $\mathcal{R}=0$ everywhere else. If we used some geometric regulator $\varepsilon$ to smooth out the cone, a term proportional to $f_{a}(n)$ would contribute to a $\log (\varepsilon / \delta)$ term, but would have no effect on the $\log (R / \delta)$ one.
} 
where the dots refer to terms which will produce logarithmic contributions in $\delta$ (but never quadratically logarithmic). Comparing this expression with eq. (2.53), we observe that the IR scale which should appear weighting $\delta$ inside the logarithm actually depends on the value of the radial coordinate $r$, i.e. it is not a fixed scale which we can factor out, and its presence has an impact in the universal coefficient. The radial integral in eq. (2.53) yields

$$
-\int_{\delta}^{R} \frac{d r}{r} \log \delta=\log ^{2} \delta+\ldots
$$

This is one of the contributions that appear in eq. (2.57), but there is another one given by

$$
\int_{\delta}^{R} \frac{d r}{r} \log r=\frac{1}{2} \log ^{2} R-\frac{1}{2} \log ^{2} \delta
$$

namely, there is an additional contribution proportional to $\log ^{2} \delta$ coming from this, which must be taken into account. Combined with the first, it effectively multiplies the answer one would naively obtain from eq. (2.53) by a factor $1 / 2$.

Taking this into account, we are left with

$$
S_{n}^{\text {univ }}=-f_{b}(n) \log ^{2} \delta\left[\frac{\cos ^{2} \theta_{0}\left(1+e^{\prime 2}\right)}{2 \pi \sin \theta_{0}}\right] \int_{0}^{\pi / 2}\left[\frac{\left(1+e^{\prime 2} \sin ^{2} \theta_{0} \cos ^{2} \phi\right)^{-1 / 2} d \phi}{\left(1+e^{\prime 2} \cos ^{2} \phi\right)^{5 / 2}}\right] .
$$

At this point, one can weight $\delta$ by any of the IR scales $R$ or $z_{0}$, depending on whether we cutoff the cone at some radial distance, or at some height $z_{0} \cdot{ }^{15}$ The choice does not alter the result for the quadratically logarithmic universal term.

We can write then ${ }^{16}$

$$
S_{n}^{\text {univ }}=-a_{n}^{(4)}\left(e^{\prime}, \theta_{0}\right) \log ^{2}(R / \delta), \quad \text { where } \quad a_{n}^{(4)}\left(e^{\prime}, \theta_{0}\right)=\frac{1}{4} f_{b}(n) \gamma\left(e^{\prime}, \theta_{0}\right),
$$

and where we defined

$$
\gamma\left(e^{\prime}, \theta_{0}\right)=\frac{2 \cos ^{2} \theta_{0}\left(1+e^{\prime 2}\right)}{\pi \sin \theta_{0}} \int_{0}^{\frac{\pi}{2}} \frac{\left(1+e^{\prime 2} \sin ^{2} \theta_{0} \cos ^{2} \phi\right)^{-1 / 2}}{\left(1+e^{\prime 2} \cos ^{2} \phi\right)^{5 / 2}} d \phi .
$$

Note that for $e^{\prime}=0$, this reduces to

$$
\gamma\left(0, \theta_{0} \equiv \Omega\right)=\frac{\cos ^{2} \Omega}{\sin \Omega}
$$

and we recover the well-known result for the circular cones. In appendix $\mathrm{C}$ we show that this function generalizes in a simple way to circular hypercones in arbitrary even dimensions.

\footnotetext{
${ }^{15}$ As opposed to the circular cones case, cutting off an elliptic cone at a fixed value of the radial coordinate $r=R$ is inequivalent from cutting it off at some constant height $z=z_{0}$. In the first case, different values of $\phi$ correspond to different heights $z(\phi, R)=R \cos \theta_{0} \sqrt{1-\left(1-\kappa^{2}\right) \sin ^{2} \phi}$ for a given $R$. Hence, if we choose to cut off our cones at a fixed $r=R$, the corresponding IR boundary will be somewhat curly — see figure 2 . Alternatively, we can cut off the cones at some fixed height $z=z_{0}$, which in terms of the radial coordinate $r$ translates into integrating up to $R\left(z_{0}, \phi\right)=z_{0} /\left(\cos \theta_{0} \sqrt{1-\left(1-\kappa^{2}\right) \sin ^{2} \phi}\right)$ instead. In any case, the coefficient of the Rényi entropy universal contribution is not affected by this choice of IR cutoff.

${ }^{16}$ Observe that $\log ^{2}(R / \delta)=(\log R-\log \delta)^{2}=+\log ^{2} \delta+$ subleading.
} 
As we mentioned earlier, we could have chosen to present $\gamma\left(e^{\prime}, \theta_{0}\right)$ in terms of some other opening angle, defined by the intersection of the cone surface with a different plane containing its axis. For instance, we can express the above function in terms of $\tilde{\theta}_{0}$, as defined in eq. (2.46). ${ }^{17}$

It is possible to express $\gamma\left(e^{\prime}, \theta_{0}\right)$ in terms of known functions. The result can be written as

$$
\begin{aligned}
\gamma\left(e^{\prime}, \theta_{0}\right)= & \frac{2}{3 \pi \sin \theta_{0} \cos ^{2} \theta_{0} \alpha_{1} \alpha_{2}^{1 / 2}} \times\left\{\cos ^{2} \theta_{0}\left(e^{\prime 2} \alpha_{2}+3 \cos ^{2} \theta_{0}\right) \cdot K\left[1-\frac{\alpha_{1}}{\alpha_{2}}\right]\right. \\
& \left.-2 \alpha_{3} i\left[\alpha_{2} \cdot E\left[\frac{\alpha_{1}}{\alpha_{2}}\right]-\left(\alpha_{1} \alpha_{2}\right)^{1 / 2} \cdot E\left[\frac{\alpha_{2}}{\alpha_{1}}\right]+e^{\prime 2}\left(\frac{\alpha_{2}}{\alpha_{1}}\right)^{1 / 2} \cos ^{2} \theta_{0} \cdot K\left[\frac{\alpha_{2}}{\alpha_{1}}\right]\right]\right\},
\end{aligned}
$$

where we defined

$$
\alpha_{1} \equiv 1+e^{\prime 2}, \quad \alpha_{2} \equiv 1+e^{\prime 2} \sin ^{2} \theta_{0}, \quad \alpha_{3} \equiv 2+e^{\prime 2}-\left(3+2 e^{\prime 2}\right) \sin ^{2} \theta_{0},
$$

and where $K[x]$ and $E[x]$ are the complete elliptic integrals of the first and second kind, respectively. The expression in brackets in the second line is purely imaginary, so $j\left(e^{\prime}, \theta_{0}\right)$ is real for all physical values of $e^{\prime}$ and $\theta_{0}$.

Expansions around $\theta_{0}=\pi / 2$ and $\theta_{0}=0$ can be easily performed. The result for the first reads

$$
\begin{aligned}
\gamma\left(e^{\prime}, \theta_{0}\right)= & +\frac{8\left(1+e^{\prime 2}\right)+3 e^{\prime 4}}{8\left(1+e^{\prime 2}\right)^{3 / 2}}\left(\theta_{0}-\frac{\pi}{2}\right)^{2}+\frac{16+56 e^{\prime 2}+34 e^{\prime 4}+9 e^{\prime 6}}{96\left(1+e^{\prime 2}\right)^{5 / 2}}\left(\theta_{0}-\frac{\pi}{2}\right)^{4} \\
& +\mathcal{O}\left(\theta_{0}-\frac{\pi}{2}\right)^{6}
\end{aligned}
$$

The second one yields a leading divergent term of order $1 / \theta_{0}$, just like in the circular-cones case. The coefficient can be obtained straightforwardly from eq. (2.64) as a function of $e^{\prime}$, but it is not particularly illuminating. At leading order in $e^{\prime}$, one finds

$$
\gamma\left(e^{\prime}, \theta_{0}\right)=\left[1-\frac{e^{\prime 2}}{4}+\mathcal{O}\left(e^{\prime 4}\right)\right] \frac{1}{\theta_{0}}+\mathcal{O}\left(\theta_{0}\right)
$$

For large values of the eccentricty, $\gamma\left(e^{\prime}, \theta_{0}\right)$ diverges linearly with $e^{\prime}$. For small $e^{\prime}$, one finds, in turn,

$$
\gamma\left(e^{\prime}, \theta_{0}\right)=\frac{\cos ^{2} \theta_{0}}{\sin \theta_{0}}\left[1-\frac{3-\cos \left(2 \theta_{0}\right)}{8} e^{\prime 2}+\frac{283-92 \cos \left(2 \theta_{0}\right)+9 \cos \left(4 \theta_{0}\right)}{512} e^{\prime 4}+\mathcal{O}\left(e^{\prime 6}\right)\right] .
$$

Since the coefficient of the quadratic term is always negative, circular cones locally maximize $\gamma$ for fixed $\theta_{0}$. A more meaningful comparison of the whole Rényi entropy can be

\footnotetext{
${ }^{17}$ Since such opening angle is defined in the direction of the semi-major axis, $b$, our result for $\gamma\left(e^{\prime}, \theta_{0}\right)$ should take exactly the same functional form when expressed in terms of $\tilde{\theta}_{0}$ and $\tilde{e}^{\prime}$, where $\left(\tilde{e}^{\prime}\right)^{2}=-e^{\prime 2} /[1+$ $\left.e^{\prime 2}\right]$, namely, the 'eccentricity' one would obtain by flipping $a \leftrightarrow b$ in eq. (2.47). It is a straightforward excercise to show that $\gamma\left(e^{\prime}, \theta_{0}\right)$ indeed satisfies this symmetry property.
} 
performed by fixing the lateral area of the cones, instead of $\theta_{0}$, since in that case the arealaw contribution is the same. In appendix B we show that when we fix the latera area of the cones, the quadratic term in eq. (2.68) conspires to disappear, the coefficient of the quartic one being always positive. Since $\gamma$ contributes with a minus sign to $S_{n}$, the cones that maximize the Rényi entropy within the family of elliptic cones are the circular ones.

\subsubsection{Free fields}

As we have seen, for free fields we need to characterize the boundary of the intersection of the conical entangling region with $\mathbb{S}^{2}$. For circular cones, this is a $\mathbb{S}^{1}$, as shown in figure 1. The embedding of the $\mathbb{S}^{1}$ on $\mathbb{S}^{2}$ is given simply by $\theta=\Omega$. Then, the normal vector is given by $n=\partial_{\theta}$, and the induced metric is $d s_{\mathbb{S}^{1}}^{2}=\sin ^{2} \Omega d \phi$, so $\sqrt{h}=\sin \Omega$. The only nonvanishing component of the extrinsic curvature is given by

$$
k_{\phi \phi}=\frac{\partial_{\theta} g_{\phi \phi}}{2}=\sin \Omega \cos \Omega,
$$

so we find

$$
\int_{\gamma} k^{2}=\int_{0}^{2 \pi} d \phi \sqrt{h}\left(h^{\phi \phi} k_{\phi \phi}\right)^{2}=\frac{2 \pi \cos ^{2} \Omega}{\sin \Omega}
$$

and hence

$$
\left.S_{n}\right|_{\log ^{2}}=-\frac{1}{4} f_{b}(n) \frac{\cos ^{2} \Omega}{\sin \Omega} \log ^{2} \delta .
$$

which precisely agrees with the angular dependence expected for a conical entangling region for general CFTs in eq. (2.61) and eq. (2.63).

We can readily verify that this also works for elliptic cones. In that case, the metric on the unit round $\mathbb{S}^{2}$ in sphero-conal coordinates reads

$$
d s_{\mathbb{S}^{2}}^{2}=\left[\frac{\kappa^{2} \sin ^{2} \theta+\left(1-\kappa^{2}\right) \cos ^{2} \phi}{1-\kappa^{2} \cos ^{2} \theta}\right] d \theta^{2}+\left[\frac{\kappa^{2} \sin ^{2} \theta+\left(1-\kappa^{2}\right) \cos ^{2} \phi}{1-\left(1-\kappa^{2}\right) \sin ^{2} \phi}\right] d \phi^{2} .
$$

The induced metric on the intersection of the elliptic cone of semi-opening angle $\theta_{0}$ with the $\mathbb{S}^{2}$ is given by

$$
d s_{h}^{2}=\left[\frac{\kappa^{2} \sin ^{2} \theta_{0}+\left(1-\kappa^{2}\right) \cos ^{2} \phi}{1-\left(1-\kappa^{2}\right) \sin ^{2} \phi}\right] d \phi^{2} .
$$

The only non-vanishing component of the extrinsic curvature associated to the normal vector $n=\frac{1}{\sqrt{g_{\theta \theta}}} \partial_{\theta}$ reads

$$
k_{\phi \phi}=\frac{\partial_{\theta} g_{\phi \phi}}{2 \sqrt{g_{\theta \theta}}}=\frac{\kappa^{2} \sin \theta_{0} \cos \theta_{0} \sqrt{1-\kappa^{2} \cos ^{2} \theta_{0}}}{\sqrt{\kappa^{2} \sin ^{2} \theta_{0}+\left(1-\kappa^{2}\right) \cos ^{2} \phi}\left(1-\left(1-\kappa^{2}\right) \sin ^{2} \phi\right)} .
$$

Then,

$$
k^{2}=\left(h^{\phi \phi} k_{\phi \phi}\right)^{2}=\frac{\kappa^{4} \sin ^{2} \theta_{0} \cos ^{2} \theta_{0}\left(1-\kappa^{2} \cos ^{2} \theta_{0}\right)}{\left(\kappa^{2} \sin ^{2} \theta_{0}+\left(1-\kappa^{2}\right) \cos ^{2} \phi\right)^{3}} .
$$

Plugging this in eq. (2.28) along with the induced metric determinant coming from eq. (2.73), we are left with an angular integral and a dependence on $\theta_{0}$ which is again identical to the one found using Solodukhin's formula eq. (2.60), so the final result is again given by eq. (2.61) and eq. (2.64), as it should. 


\subsection{Higher dimensions}

As we argued above, the coefficient $c_{n,-1}$ in the high-mass expansion eq. (2.23) is forced to vanish for odd-dimensional CFTs. Hence, the Rényi entropy for conical entangling regions

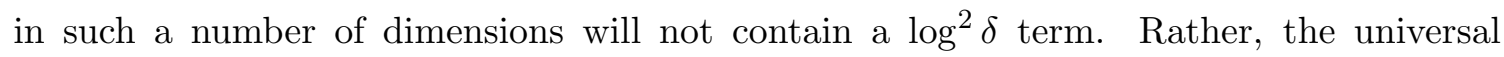
contribution will be logarithmic, the corresponding coefficient having a highly non-local origin. In the case of five-dimensional theories, this structure was explicitly verified for holographic theories dual to Einstein and Gauss-Bonnet gravities in [25].

On the other hand, for even dimensions larger than four, the $\operatorname{local} \log ^{2} \delta$ term will be present for conical regions. In appendix $\mathrm{C}$, we show that the dependence of the universal function $a_{n}^{(4)}(\Omega)$ on the opening angle generalizes in a simple way to arbitrarily high even dimensions. Namely, we argue that the universal contribution to the Rényi entropy of right circular (hyper)cones is given, for general CFTs, by the simple formula

$$
S_{n}^{\text {univ }}=(-1)^{\frac{d-2}{2}} a_{n}^{(d)}(\Omega) \log ^{2}\left(\frac{R}{\delta}\right), \quad \text { with } \quad a_{n}^{(d)}(\Omega)=\frac{\cos ^{2} \Omega}{\sin \Omega} \sum_{j=0}^{\frac{d-4}{2}}\left[\gamma_{j, n}^{(d)} \cos (2 j \Omega)\right],
$$

where the only information about the underlying theory appears through the coefficients $\gamma_{j, n}^{(d)}$, which will be related to the Rényi entropy generalizations of the traceanomaly charges.

\section{$3 \quad$ Wedge entanglement versus corner entanglement}

In this section we study the entanglement entropy of a region bounded by a wedge in $(3+1)$ dimensional CFTs. We have already encountered wegdges of opening angle $\Omega=\pi / 2$ in our analysis of the entanglement entropy of trihedral corners using the EMI. Here, we begin by analyzing the wedge contribution in the nearly smooth limit, $\Omega \simeq \pi$, for general CFTs. We then consider the calculation for free fields using dimensional reduction. We complement the free scalar calculation in [57] with an analysis of the emergence of $f(\Omega)$ from $a(\Omega)$ for general free fields (scalars and fermions). We confirm that the angular dependence of $f(\Omega)$ is indeed a universal quantity given by $a(\Omega)$, which is the $(2+1)$-dimensional corner function of the corresponding lower dimensional free theory. We also illustrate how the nonuniversal character of the overall factor is connected to the different possible choices of regulators along the transverse and corner directions. Finally, we show that, contrary to a previous claim made in [57], the wedge and corner functions do differ for holographic theories dual to Einstein gravity in the bulk. This suggests that the relation between the wedge and the lower dimensional corner function does not hold for interacting theories.

The setup is the following. In the wedge case, the entangling region at some fixed time slice corresponds to the set $\{(r, \phi, z)$ such that $0 \leq r<\infty, 0 \leq z<\infty, 0 \leq \phi \leq \Omega\}$ in cylindrical coordinates. The entanglement entropy takes the form in eq. (1.7), namely:

$$
S_{\mathrm{EE}}=b_{2} \frac{H^{2}}{\delta^{2}}-f(\Omega) \frac{H}{\delta}+\mathcal{O}\left(\delta^{0}\right),
$$

where $H$ and $\delta$ are IR and UV cutoffs respectively, $b_{2}$ is a nonuniversal constant, and $f(\Omega)$ is a function of the wedge opening angle whose overall normalization depends on the 
UV cutoff. The analogous entangling region bounded by the corner in one dimension less is given in polar coordinates by $\{(r, \phi)$ such that: $0 \leq r<\infty, 0 \leq \phi \leq \Omega\}$. There, the entanglement entropy reads

$$
S_{\mathrm{EE}}=b_{1} \frac{H}{\delta}-a(\Omega) \log \left(\frac{H}{\delta}\right)+\mathcal{O}\left(\delta^{0}\right),
$$

where $b_{1}$ is some other nonuniversal constant, and $a(\Omega)$ is a cutoff-independent function of the opening angle.

\subsection{General CFTs in the nearly smooth limit}

To obtain the wedge function in the nearly smooth limit, $\Omega \simeq \pi$, we will employ the 2 nd order entanglement susceptibility, $\chi^{(2)}{ }^{18}$ This was for example used [47] to obtain the $d=3$ corner function of a general CFT in the nearly smooth limit $[26,46]$ :

$$
a(\Omega)=\frac{\pi^{2} C_{T}}{24}(\pi-\Omega)^{2}+\ldots
$$

The idea is to consider how the entanglement entropy changes as a function of a small deformation $A \rightarrow A+\delta A$ :

$$
\begin{aligned}
S_{\mathrm{EE}}(A+\delta A)= & S_{\mathrm{EE}}(A)+\int_{\partial A} d^{d-2} \mathbf{r} \chi^{(1)}(\mathbf{r}) \zeta(\mathbf{r}) \\
& +\frac{1}{2 !} \int_{\partial A} d^{d-2} \mathbf{r} \int_{\partial A} d^{d-2} \mathbf{r}^{\prime} \chi^{(2)}\left(\mathbf{r}, \mathbf{r}^{\prime}\right) \zeta(\mathbf{r}) \zeta\left(\mathbf{r}^{\prime}\right)+\cdots
\end{aligned}
$$

The deformation $\delta A$ is defined by sending a point $\mathbf{r}$ on $\partial A$ to $\zeta(\mathbf{r}) \mathbf{n}(\mathbf{r})$, where $\mathbf{n}(\mathbf{r})$ is the unit normal at $\mathbf{r}$ and $\zeta(\mathbf{r})$ is taken to be small. We shall consider the case where the state is the vacuum of a CFT, and $A$ is the half-space at a fixed time slice. Since we are working with a pure state, the odd susceptibilities such as $\chi^{(1)}$ vanish. The first contribution will come from $\chi^{(2)}$, where [47]

$$
\chi^{(2)}\left(\mathbf{r}-\mathbf{r}^{\prime}\right)=-\frac{2 \pi^{2} C_{T}}{d+1} \frac{1}{\left|\mathbf{r}-\mathbf{r}^{\prime}\right|^{2(d-1)}},
$$

is the non-local or universal part of the susceptibility, which is controlled by the 2-point function coefficient of the stress tensor, $C_{T}$.

Setting $d=4$, let us parametrize $A$ as the half-space $z<0$, where the spatial coordinates are $\mathbf{r}=(x, y, z)$. We then choose the following deformation

$$
\zeta(\mathbf{r})=\Theta(x) x \tan \alpha
$$

where $\Theta(x)$ is the Heaviside step function. This introduces a wedge of angle $\Omega=\pi-\alpha$, where $\alpha$ is taken to be small. Using eq. (3.4), the variation of the entanglement entropy reads

$$
\delta S_{\mathrm{EE}}=-\frac{\pi^{2} C_{T} \tan ^{2} \alpha}{5} \int_{0}^{\infty} d x d x^{\prime} \int_{-\infty}^{\infty} d y d y^{\prime} \frac{x x^{\prime}}{\left(\left(x-x^{\prime}\right)^{2}+\left(y-y^{\prime}\right)^{2}\right)^{3}} .
$$

\footnotetext{
${ }^{18}$ This quantity is sometimes called "entanglement density", but we shall not use this convention.
} 
We first perform the $x^{\prime}$ integral; the integrand becomes

$$
g(x, \Delta)=\frac{x\left(3 \pi x \operatorname{sgn}(\Delta)+2 \Delta\left(\frac{x^{2}}{\Delta^{2}+x^{2}}+2\right)+6 x \tan ^{-1}\left(\frac{x}{\Delta}\right)\right)}{16 \Delta^{5}},
$$

where we defined $\Delta=y-y^{\prime}$. Before performing the $x$-integral, let us perform a large- $x$ expansion to isolate the IR divergent term:

$$
g(x, \Delta)=\frac{3 \pi x^{2} \operatorname{sgn}(\Delta)}{8 \Delta^{5}}+\frac{1}{20 x^{3}}+O\left(1 / x^{4}\right) .
$$

We can then separate the IR divergent piece:

$$
\begin{aligned}
\int d x f(x, \Delta) & =\int_{0}^{\infty} d x\left[g(x, \Delta)-\frac{3 \pi x^{2} \operatorname{sgn}(\Delta)}{8 \Delta^{5}}\right]+\int_{0}^{H} d x \frac{3 \pi x^{2} \operatorname{sgn}(\Delta)}{8 \Delta^{5}} \\
& =\frac{1}{24 \Delta^{2}}+\frac{\pi H^{3} \operatorname{sgn}(\Delta)}{8 \Delta^{5}}
\end{aligned}
$$

where $H$ is the IR cutoff. We now perform the $y^{\prime}$ integral using a splitting regularization: $\int_{-\infty}^{\infty} d y^{\prime}=\int_{-\infty}^{y-\delta} d y^{\prime}+\int_{y+\delta}^{\infty} d y^{\prime}$. Finally, the $y$ integral can beformed with UV an IR cutoffs, and we obtain:

$$
\delta S_{\mathrm{EE}}=-\frac{\pi^{2} C_{T}(\Omega-\pi)^{2}}{60} \frac{H}{\delta}+\cdots
$$

where the dots represent not only subleading terms in $\delta$, but also $1 / \delta^{4}$ and $1 / \delta^{3}$ divergences. These two unphysical terms result from our regularization scheme, and should be discarded. They do not influence the wedge contribution $\propto H / \delta$. We thus see that in the nearly smooth limit, the wedge function is:

$$
f(\Omega)=\frac{\pi^{2} C_{T}}{60}(\Omega-\pi)^{2},
$$

up to an overall regularization-dependent prefactor. In this limit, the wedge function behaves in a very similar way to the corner function $a(\Omega)$ in one lower dimension, eq. (3.3). This result also holds for the Rényi entropies, but with the replacement of $C_{T}$ by $f_{b}(n)$ (up to an unimportant prefactor) [54].

\subsection{Free fields}

Certain contributions to the entanglement entropy of $d$-dimensional free-field theories can be obtained from others corresponding to $(d-1)$-dimensional contributions [17,66]. This is the case, in particular, when the entangling region $W$ takes the form of a direct product, $W=C \times \mathbb{R}$, where $C$ is some $(d-1)$-dimensional set. In this situation, one would usually cutoff the extra dimension, which we parametrize here by $z$, at some finite distance $L$ to avoid an IR-divergent result. The idea here is to compactify $z$ imposing periodic boundary conditions, $z=z+L$, and then decompose the corresponding $d$-dimensional field into its Fourier modes along that direction. This reduces the problem to a $(d-1)$-dimensional one. 


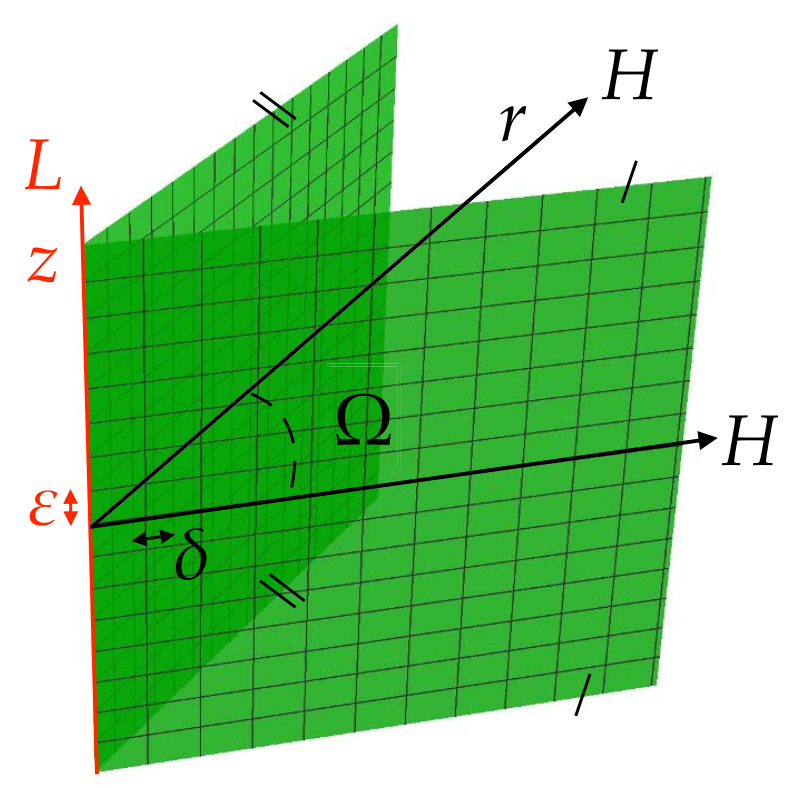

Figure 3. We plot a wedge-shaped entangling surface of opening angle $\Omega$ and a corner region in one dimension less resulting from its dimensional reduction. While the wedge contribution to the entanglement entropy $-f(\Omega) H / \delta$ gets polluted by the presence of inequivalent regulators along the radial and transverse directions - see eq. (3.20) - the dependence on the opening angle is well-defined.

Starting with a mass- $m$ free field in $d$-dimensions, the problem is mapped to the one of infinitely many $(d-1)$-dimensional independent fields of masses

$$
M_{k}^{2}=m^{2}+p_{k}^{2},
$$

where $p_{k} \equiv(2 \pi k / L)$ is the momentum of the $k$-th mode along $z$. Then, the entanglement entropy for $W$ in the $d$-dimensional theory can be obtained by summing over all $(d-1)$ dimensional entropies corresponding to the entangling region $C$. In the large- $L$ limit, the sum can be converted into an integral of the form $[17,17,66]$

$$
S_{\mathrm{EE}}(W)=\frac{c_{\mathrm{f}} L}{\pi} \int^{1 / \epsilon} d p S_{\mathrm{EE}}\left(C, \sqrt{m^{2}+p^{2}}\right)
$$

where the extensivity of $S_{\mathrm{EE}}(W)$ on $L$ is manifest, and where ${ }^{19}$

$$
c_{\mathrm{f}}= \begin{cases}1 & \text { for a scalar } \\ 2^{\left\lfloor\frac{d}{2}\right\rfloor} / 2^{\left\lfloor\frac{d-1}{2}\right\rfloor} & \text { for a (Dirac) fermion } .\end{cases}
$$

Note also that we have introduced a spatial cutoff $\epsilon$, so that we do not consider infinitely massive modes, but only those with energies smaller than $1 / \epsilon$.

\footnotetext{
${ }^{19}$ For the fermion, $c_{\mathrm{f}}$ is the quotient between the dimensions of the spinorial spaces of $d$ and $(d-1)$ dimensions, respectively. When $d$ is an odd number, $c_{\mathrm{f}}=1$, whereas for even $d, c_{\mathrm{f}}=2$.
} 
Of course, in the case at hand, $W$ is the wedge in $(3+1)$-dimensions, and $C$ is the corner in $(2+1)$ - see figure 3 . For the latter, the entanglement entropy for a massive field takes the form

$$
S_{\mathrm{EE}}(C, m)=b_{1} \frac{H}{\delta}+b_{0} m H+a(\Omega) \log (m \delta)+\ldots
$$

in the large-mass limit. Note that the scale weighting the UV cutoff $\delta$ along the corner directions in the logarithmic contribution controlled by the universal function $a(\Omega)$ is $1 / m$ rather than the IR cutoff $H$, which reflects the local character of this term.

Plugging this expression in eq. (3.15), performing the integrals and considering the massless limit, we are left with

$$
S_{\mathrm{EE}}(W)=\frac{b_{1} c_{\mathrm{f}}}{\pi} \frac{H L}{\epsilon \delta}+\frac{b_{0} c_{\mathrm{f}}}{2 \pi} \frac{L H}{\epsilon^{2}}-\frac{a(\Omega) c_{\mathrm{f}}}{\pi} \frac{L}{\epsilon}\left[1-\log \left(\frac{\delta}{\epsilon}\right)\right] .
$$

This is an interesting result. First, we observe that the first two terms, coming from the area-law like term in eq. (3.17) and $m H$, both contribute to the area law in $S_{\mathrm{EE}}(W)$. This is manifest if we write the UV cutoff along the $z$ direction in terms of the one along the corner radial directions, $\epsilon=\alpha_{\mathrm{UV}} \delta$, for some $\mathcal{O}(1)$ constant $\alpha$. We can also relate the IR cutoffs $L$ and $H, L=\alpha_{\mathrm{IR}} H$. Then, we obtain

$$
S_{\mathrm{EE}}(W)=b_{2} \frac{H^{2}}{\delta^{2}}-\frac{a(\Omega) c_{\mathrm{f}}}{\pi} \frac{H}{\delta}\left[1+\log \left(\alpha_{\mathrm{UV}}\right)\right] \alpha_{\mathrm{IR}},
$$

where $b_{2}$ is some nonuniversal constant. This precisely takes the form in eq. (3.1) expected for general CFTs. We observe that the function $f(\Omega)$ is indeed related to the corner function $a(\Omega)$ through

$$
f(\Omega) \propto a(\Omega)\left[1+\log \left(\alpha_{\mathrm{UV}}\right)\right] \alpha_{\mathrm{IR}} .
$$

There is no physical reason to prefer, say, $\alpha_{\mathrm{IR}}=\pi / c_{\mathrm{f}}, \alpha_{\mathrm{UV}}=1$ over any other choice, which illustrates the nonuniversal character of the overall factor in $f(\Omega)$ and how this is polluted by the different choices of regulators. The angular dependence, however, is physically meaningful, and inherited from the corner one, as observed in [57] in the particular case of a scalar field. Note also that the above connection extends straightforwardly to general Rényi entropies.

Naturally, the dimensional reduction performed here is exclusively valid for free scalars and fermions. It is nonetheless tempting to speculate with the possibility that $a(\Omega)$ and $f(\Omega)$ may be connected in a similar fashion for a larger family of CFTs [57]. As we show in the following subsection, this is not the case in general, as the angular dependence of both functions is in fact different for holographic CFTs dual to Einstein gravity in the bulk.

\subsection{Holography}

Consider now holographic theories dual to Einstein gravity. The bulk action is given by

$$
I=\frac{1}{16 \pi G} \int d^{(d+1)} x \sqrt{|g|}\left[\frac{d(d-1)}{L^{2}}+R\right]
$$



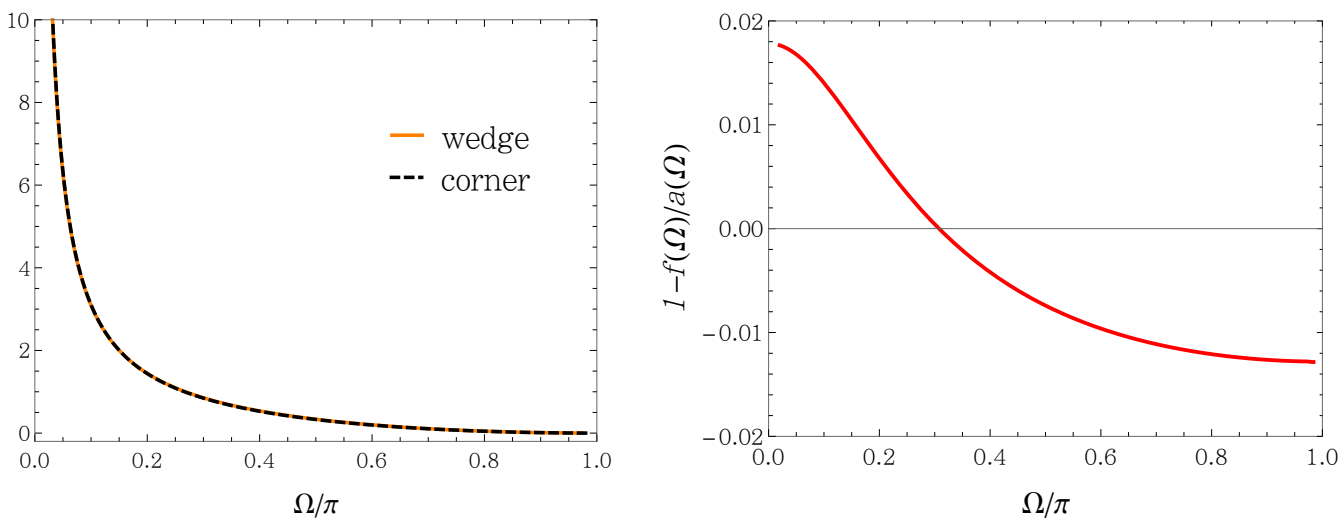

Figure 4. (Left) We plot the wedge (orange) and corner (black dashed) functions $f(\Omega)$ and $a(\Omega)$ normalized so as to make them fall approximately on top of each other, as observed in [57]. (Right) We plot $1-f(\Omega) / a(\Omega)$. As we can see, both functions are in fact slightly different from each other.

where $G$ is Newton's constant, and the cosmological constant length-scale $L$ coincides with the $\operatorname{AdS}_{(d+1)}$ radius. Then, the result for the universal function $a(\Omega)$ appearing in the entanglement entropy of a corner region in $d=3$, computed using the Ryu-Takayanagi prescription $[72,73]$, is given by $[24,74]$

$$
a(\Omega)=\frac{L^{2}}{2 G} \int_{g_{0}}^{\infty} d g \frac{g}{\sqrt{g^{2}-g_{0}^{2}}}\left[1-\sqrt{\frac{1+g^{2}}{1+g_{0}^{2}+g^{2}}}\right]
$$

where $g_{0}(\Omega)$ is an implicit function of the opening angle, namely

$$
\Omega=\int_{g_{0}}^{\infty} d g \frac{2}{g \sqrt{\left(1+g^{2}\right)\left(\frac{g^{2}\left(1+g^{2}\right)}{g_{0}^{2}\left(1+g_{0}^{2}\right)}-1\right)}}
$$

On the other hand, the holographic result for the wedge region $f(\Omega)$ reads $[25,57]$

$$
f(\Omega)=\frac{L^{3}}{2 G}\left[g_{0}-\int_{g_{0}}^{\infty} d g\left(\frac{g\left(1+g^{2}\right)}{\sqrt{g^{2}\left(1+g^{2}\right)^{2}-g_{0}^{2}\left(1+g_{0}^{2}\right)^{2}}}-1\right)\right],
$$

where $g_{0}$ is related to the wedge opening angle through

$$
\Omega=\int_{g_{0}}^{\infty} d g \frac{2}{g \sqrt{\left(1+g^{2}\right)\left(\frac{g^{2}\left(1+g^{2}\right)^{2}}{g_{0}^{2}\left(1+g_{0}^{2}\right)^{2}}-1\right)}} .
$$

As mentioned earlier, in [57] it was observed that — at least within the numerical resolution considered - the dependence of both functions on the corresponding opening angles appears to be actually identical, i.e. $f(\Omega)=a(\Omega)$ up to a global factor. As we show here, the claim is actually not correct in the holographic case. 
In the $\Omega \rightarrow 0$ and $\Omega \rightarrow \pi$ limits, corresponding to very sharp and almost smooth corner/wedges, respectively, it is possible to show that $a(\Omega)$ and $f(\Omega)$ behave as ${ }^{20}$

$$
\begin{aligned}
& a(\Omega) \stackrel{\Omega \rightarrow 0}{=} \frac{\kappa}{\Omega}+\ldots, \quad a(\Omega) \stackrel{\Omega \rightarrow \pi}{=} \sigma \cdot(\Omega-\pi)^{2}+\ldots, \\
& f(\Omega) \stackrel{\Omega \rightarrow 0}{=} \frac{\tilde{\kappa}}{\Omega}+\ldots, \quad f(\Omega) \stackrel{\Omega \rightarrow \pi}{=} \tilde{\sigma} \cdot(\Omega-\pi)^{2}+\ldots,
\end{aligned}
$$

where the dots stand for subleading contributions, and where

$$
\kappa=\Gamma\left(\frac{3}{4}\right)^{4} \frac{L^{2}}{2 \pi G}, \quad \sigma=\frac{L^{2}}{8 \pi G}, \quad \tilde{\kappa}=\frac{2^{2 / 3} \pi^{3 / 2} \Gamma\left(\frac{5}{6}\right) L^{3}}{\Gamma\left(\frac{1}{6}\right)^{2} G}, \quad \tilde{\sigma}=\frac{3 \pi L^{3}}{256 G} .
$$

As we mentioned earlier, the overall constant in $f(\Omega)$ is not well defined, so the values of $\tilde{\kappa}$ and $\tilde{\sigma}$ are not meaningful by themselves. However, the ratio $\tilde{\kappa} / \tilde{\sigma}$ is in principle a meaningful quantity. If the claim in [57] were true, such ratio should agree with the one corresponding to the corner function. We find, however

$$
\frac{\kappa}{\sigma}=4 \Gamma\left(\frac{3}{4}\right)^{4} \simeq 9.0198, \quad \frac{\tilde{\kappa}}{\tilde{\sigma}}=\frac{2^{2 / 3} 256 \sqrt{\pi} \Gamma\left(\frac{5}{6}\right)}{3 \Gamma\left(\frac{1}{6}\right)^{2}} \simeq 8.7469,
$$

which is close, but obviously different. This discrepancy can also be observed by plotting $[1-f(\Omega) / a(\Omega)]$, as we have done in figure 4 . Remarkably, the overall factor can be chosen so that $f(\Omega)$ and $a(\Omega)$ differ by less than $\sim 2 \%$ for each value of $\Omega$.

\section{Singular geometries versus entanglement divergences}

In this section we analyze the interplay between singular entangling surfaces, and the structure of divergences of entanglement entropies and mutual information. First we show that, contrary to the usual expectations, mutual information $I(A, B)$ does not necessarily become divergent in the limit when $A$ and $B$ have contact points. In particular, whenever the contact is through a sufficiently sharp corner (anything sharper than a straight corner works), $I(A, B)$ remains finite. Then, we consider the entanglement entropy of curved corners. We provide examples of singular regions which do not change the structure of divergences/universal terms with respect to the smooth case, and also how new divergences can appear when the corners become sufficiently sharp.

In order to extract our conclusions, we make use of the Extensive Mutual Information model again - see eq. (2.31). In that model, the mutual information between two entangling regions $A$ and $B$, can be written in terms of a simple local integral as

$$
I^{\mathrm{EMI}}(A, B)=-2 \kappa \int_{\partial A} d \mathbf{r} \int_{\partial B} d \mathbf{r}^{\prime} \frac{\mathbf{n} \cdot \mathbf{n}^{\prime}}{\left|\mathbf{r}-\mathbf{r}^{\prime}\right|^{2(d-2)}} .
$$

${ }^{20}$ Observe that for $\Omega \rightarrow \pi$, the Einstein gravity result for $f(\Omega)$ can be written in terms of $C_{T}$ as

$$
f(\Omega)=\frac{3 \pi^{4} C_{T}}{1280}(\Omega-\pi)^{2}+\ldots
$$

where we used $C_{T}=5 L^{3} /\left(\pi^{3} G\right)$. This disagrees with eq. (3.13), which is not very surprising given that $f(\Omega)$ is only defined up to an overall regulator-dependent coefficient. 

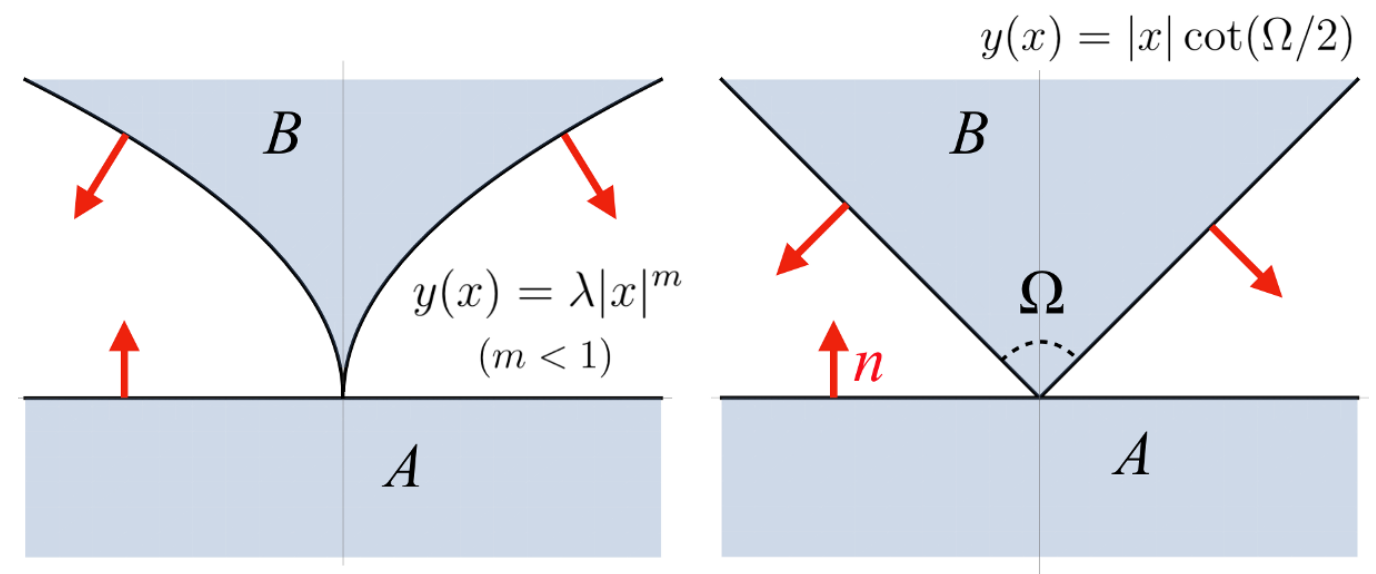

Figure 5. We show two entangling regions on a time slice of a three-dimensional theory corresponding, respectively, to a half plane and a region characterized by a corner defined by the regions $y(x) \geq \lambda|x|^{m}, \lambda \geq 0$ (left) and $y(x) \geq|x| \cot (\Omega / 2)$ (right). The red arrows correspond to normal vectors to the entangling surfaces. For the straight corner, the mutual information $I(A, B)$ diverges logarithmically, eq. (4.3). Whenever $m<1$, however, the mutual information remains finite even when $A$ and $B$ touch each other at a point.

While a free fermion in two dimensions satisfies the extensivity property eq. (2.30), no explicit CFT in $d>2$ is known (at least, for the moment) to do so. Nonetheless, the expressions for the entanglement entropy and the mutual information do respect the generic features corresponding to those quantities in general dimensions. While the exact details of the different terms will depend on the particular theory under consideration, we expect our conclusions regarding the structure of divergences to hold for general CFTs.

\subsection{Finite mutual information for touching regions}

The mutual information between two regions $A$ and $B$ is typically finite. However, as we move the corresponding entangling surfaces close to each other, it is expected that $I(A, B)$ becomes divergent. For instance, if $A$ is a disk of radius $R$ and $B$ is the exterior of a circular region of radius $R+\varepsilon$ concentric with $A$, the mutual information diverges as $I(A, B) \sim R / \varepsilon$. In fact, if we have some "interior" region $A$ and some "exterior" region $B$ such that their boundaries are two parallel curves separated by a curved strip of width $\varepsilon$, one can use the mutual information corresponding to this setup to define a regulator for entanglement entropy, with $\varepsilon$ playing the role of UV cutoff [12]. Here we show that if the contact region between $A$ and $B$ is sufficiently sharp, $I(A, B)$ is still finite in the limit in which both surfaces touch at a point.

For concreteness we set $d=3$. We choose $A$ to be the lower half plane - see figure 5 . Hence, for the EMI model defined in eq. (4.1) we have $\mathbf{n}\left(\mathbf{r}^{\prime}\right)=(0,1)$ and we can write

$$
I(A, B)=2 \kappa \int_{\partial B} d \mathbf{r} n_{y} \int_{-\infty}^{+\infty} \frac{d x^{\prime}}{\left(x^{\prime}-x\right)^{2}+y^{2}}=-2 \pi \kappa \int_{\partial B} \frac{n_{y}}{y} d \mathbf{r},
$$

where we used the notation $\mathbf{n}(\mathbf{r}) \equiv\left(n_{x}, n_{y}\right)$ and $\mathbf{r} \equiv(x, y)$. Let us now choose region $B$ to be defined by $y(x) \geq \lambda|x|^{m}$, where $m>0$ and $\lambda$ has units of (length $)^{1-m}$. In the limiting 
case $m=1, \lambda \equiv \cot (\Omega / 2)$, this corresponds to a region with a straight corner of opening angle $\Omega$. For $m<1$, the corner becomes sharper, and the opposite for $m>1$. For general $m$ we have $d \mathbf{r} n_{y}=-d x$. Then, in the case of a straight corner, the result for the mutual information reads

$$
I(A, B)=4 \pi \kappa \tan (\Omega / 2) \int_{(\delta / \cot (\Omega / 2))}^{L} \frac{d x}{x}=4 \pi \kappa \tan (\Omega / 2) \log \left(\frac{L}{\delta}\right)+\mathcal{O}\left(\delta^{0}\right),
$$

which is obviously divergent as $\delta \rightarrow 0 .{ }^{21}$ Similarly, for $m \neq 1$ one finds

$$
I(A, B)=\frac{4 \pi \kappa}{\lambda} \int_{y^{-1}(\delta)}^{L} \frac{d x}{x^{m}}=\frac{4 \pi \kappa}{(1-m)}\left[\frac{L^{1-m}}{\lambda}-\frac{1}{\lambda^{\frac{1}{m}} \delta^{1-\frac{1}{m}}}\right]
$$

which is always non-negative. Note that in this expression we have introduced an IR cutoff $L$ and an UV cutoff as $\partial B$ approaches $\partial A$ a distance $\delta$ in the $y$ direction, i.e. at $x=(\delta / \lambda)^{1 / m}$. Alternatively, we can make this cutoff go to zero and instead shift the $B$ region vertically a distance $\delta$, so that $\partial B$ is defined as $y(x)=\lambda|x|^{m}+\delta$. In both cases, the net result is the appearance of a piece $I(A, B) \sim \delta^{1 / m-1}$. This term is divergent whenever $m \geq 1$, but vanishes for $m<1$. In that case, the mutual information is UV finite. While the exact coefficients will vary if we consider a different CFT, we expect this phenomenon to hold for general theories.

Note also that the divergences observed approach an area-law as $m \rightarrow \infty$. As $m$ grows, region $B$ tends to be more open, and more points in $\partial B$ become closer to points in $\partial A$ in an increasingly bigger neighborhood of the touching point. In the limit, the situation is similar to the case described above in which $\partial A$ and $\partial B$ are concentric circles, which in the $\varepsilon \rightarrow 0$ limit have an area-law divergent mutual information.

\subsection{Entanglement entropy of curved corners}

Typically, the presence of geometric singularities on the entangling surface modifies the structure of divergences - and universal terms - of entanglement/Rényi entropies. A prototypical example of this phenomenon occurs in $(2+1)$ dimensions. When the entangling surface is smooth, the Rényi entropy contains a single (constant) term in addition to the area-law one. However, if a corner of opening angle $\Omega$ is present on the surface, a new logarithmically divergent term with a universal prefactor appears - see eq. (3.2) above. As we mentioned above, conical regions in $(3+1)$ dimensions - and in contradistinction to smooth ones, for which the subleading (universal) term is logarithmic - produce universal $\log ^{2}(H / \delta)$ terms, wedges give rise to $H / \delta$ divergences, and so on. However, not all entangling surfaces containing geometric singularities modify the structure of divergences. A first case in which this does not happen was analyzed in section 2 for polyhedral vertices. There, the agreement with the order of divergence of the universal term for a smooth region was however accidental, in the sense that the corresponding universal coefficients had very different origins.

\footnotetext{
${ }^{21}$ The mutual information between two corners in $(2+1)$ dimensions was studied using the AdS/CFT correspondence [31]. However, the calculation was performed for 2 corners of equal opening angles, which is different from our setup.
} 
In this section we present genuine examples of singular entangling regions which do not modify the structure of divergences/universal terms with respect to the smooth case. We also show that in other cases, namely when the entangling region contains sufficiently sharp curved corners, new divergences appear, which approach (but never get more divergent than) an area-law in the limiting case. Again for simplicity, we restrict ourselves to curved corner regions in $(2+1)$-dimensional CFTs. ${ }^{22}$ Again, we will use the Extensive Mutual Information model to derive our conclusions.

We will consider entangling regions bounded by the curves

$$
\begin{aligned}
y(x) & =\lambda x^{m}, \quad(x>0), \\
y^{\prime}\left(x^{\prime}\right) & =-\gamma x^{\prime n}, \quad\left(x^{\prime}>0\right),
\end{aligned}
$$

where the exponents $m$ and $n$ are non-negative, and $\lambda, \gamma>0$ are positive constants. Examples are shown in figure 6 for $n=m$ and $\lambda=\gamma$ and in figure 7 for $\gamma=0$. On general grounds, there will be two kinds of contributions to $S_{\mathrm{EE}}^{\mathrm{EMI}}$ as defined in eq. (2.31). The first corresponds to the contribution coming from $\mathbf{r}$ and $\mathbf{r}^{\prime}$ lying on the same curve. We assume this to be defined by the equation $y(x)=\lambda x^{m},(x \geq 0)$. The induced metric on this curve is given by $d s_{h}^{2}=\left[1+m^{2} \lambda^{2} x^{2(m-1)}\right] d x^{2}$. We also have $\mathbf{n}(\mathbf{r})=\left(-m \lambda x^{(m-1)}, 1\right) / \sqrt{1+m^{2} \lambda^{2} x^{2(m-1)}}$, so

$$
\mathbf{n}(\mathbf{r}) \cdot \mathbf{n}\left(\mathbf{r}^{\prime}\right)=\frac{1+m^{2} \lambda^{2} x^{(m-1)} x^{\prime(m-1)}}{\sqrt{1+m^{2} \lambda^{2} x^{2(m-1)}} \sqrt{1+m^{2} \lambda^{2} x^{\prime 2(m-1)}}},
$$

and

$$
\left|\mathbf{r}-\mathbf{r}^{\prime}\right|^{2}=\left(x-x^{\prime}\right)^{2}+\lambda^{2}\left(x^{m}-x^{\prime m}\right)^{2} .
$$

Using this, we can define one possible contribution to the entanglement entropy $s_{1}(m, \lambda)$ as

$$
s_{1}(m, \lambda)=\kappa \int_{\delta}^{H} d x^{\prime}\left[\int_{0}^{x^{\prime}-\delta} d x+\int_{x^{\prime}+\delta}^{\infty} d x\right] \frac{\left[1+m^{2} \lambda^{2} x^{(m-1)} x^{\prime(m-1)}\right]}{\left(x-x^{\prime}\right)^{2}+\lambda^{2}\left(x^{m}-x^{\prime m}\right)^{2}},
$$

where we introduced UV and IR cutoffs $\delta$ and $H$. Observe that in the case of a corner formed by straight lines, $s_{1}(1, \lambda)=s_{1}(1,0)$. The second possible contribution will arise from $\mathbf{r}$ and $\mathbf{r}^{\prime}$ lying on different curves. The contribution from this situation, which must be counted twice (we can flip the labels $\mathbf{r}$ and $\mathbf{r}^{\prime}$ ) reads then

$$
s_{2}(m, \lambda ; n, \gamma)=2 \kappa \int_{\delta}^{H} d x^{\prime}\left[\int_{0}^{x^{\prime}-\delta} d x+\int_{x^{\prime}+\delta}^{\infty} d x\right] \frac{\left[-1+m n \lambda \gamma x^{(m-1)} x^{(n-1)}\right]}{\left(x-x^{\prime}\right)^{2}+\left(\lambda x^{m}+\gamma x^{\prime n}\right)^{2}} .
$$

Observe that since the normal vectors must be chosen to point outwards - this is just a convention, but once chosen, it must be respected - from the entangling region, $s_{2}(m, \lambda ; m,-\lambda)$ differs by an overall sign from $s_{1}(m, \lambda)$, for which the normal vectors both point in the same direction.

\footnotetext{
${ }^{22}$ Similar geometric configurations were considered in the context of holographic Wilson loops in [75-78].
} 
Note that both $s_{1}$ and $s_{2}$ take the form

$$
s=\int_{\delta}^{H} d x^{\prime} \int_{0}^{\infty} d x f\left(x, x^{\prime}\right)-\int_{\delta}^{H} d x^{\prime} \int_{x^{\prime}-\delta}^{x^{\prime}+\delta} d x f\left(x, x^{\prime}\right),
$$

for some function $f\left(x, x^{\prime}\right)$ in each case. With the present regularization, the dependence on the UV cutoff $\delta$ appears only through the integration limits of the integrals. Hence, it follows that

$$
\frac{\partial s}{\partial \delta}=-\int_{2 \delta}^{\infty} f(x, \delta) d x-\int_{\delta}^{H}[f(x-\delta, x)+f(x+\delta, x)] d x,
$$

which is often a much easier expression to use in practice ${ }^{23}$ when extracting the structure of divergences of $s_{1}(m, \lambda)$ and $s_{2}(m, \lambda ; n, \gamma)$. Of course, $\partial s / \partial \delta$ is blind to $\mathcal{O}\left(\delta^{0}\right)$ contributions, but not to the rest of the terms.

The entanglement entropy for various corner regions will involve linear combinations of $s_{1}$ and $s_{2}$ for different values of $m, n, \lambda$ and $\gamma$. For example, in the case of a straight corner of opening angle $\Omega$ one finds $S_{\mathrm{EE}}=2 s_{1}(1,0)+s_{2}(1,0 ; 1, \tan \Omega)$. These integrals yield

$$
\begin{aligned}
2 s_{1}(1,0) & =\frac{4 \kappa H}{\delta}-2 \kappa \log \left(\frac{H}{\delta}\right)+\mathcal{O}\left(\delta^{0}\right), \\
s_{2}(1,0 ; 1, \tan \Omega) & =-2 \kappa(\pi-\Omega) \cot \Omega \log \left(\frac{H}{\delta}\right)+\mathcal{O}\left(\delta^{0}\right) .
\end{aligned}
$$

Then, one is left with

$$
S_{\mathrm{EE}}=\frac{4 \kappa H}{\delta}-a(\Omega) \log \left(\frac{H}{\delta}\right)+\mathcal{O}\left(\delta^{0}\right), \quad \text { where } \quad a(\Omega)=2 \kappa[1+(\pi-\Omega) \cot \Omega] .
$$

This expression for the corner function $a(\Omega)$ in the EMI model was previously obtained in $[18,46,67,68]$.

Let us now consider the case of an entangling region defined by $-\lambda x^{m} \leq y(x) \leq \lambda x^{m}$, with $m>0$, as shown in figure 6 . In that situation, the entangling region contains a geometric singularity at the origin for $m>1 / 2$. For $0<m \leq 1 / 2$, however, the surface is non-singular. The result for the entanglement entropy is given in this case by $S_{\mathrm{EE}}=$ $2 s_{1}(m, \lambda)+s_{2}(m, \lambda ; m, \lambda)$. Using eq. (4.12), one can show that these two contributions behave as

$$
\begin{aligned}
2 s_{1}(m, \lambda) & =\frac{4 \kappa H}{\delta}-2 \kappa m \log \left(\frac{H}{\delta}\right)+\mathcal{O}\left(\delta^{0}\right), \\
s_{2}(m, \lambda ; m, \lambda) & =2 \kappa m \log \left(\frac{H}{\delta}\right)+\mathcal{O}\left(\delta^{0}\right),
\end{aligned}
$$

for $1 / 2<m<1$. Hence,

$$
S_{\mathrm{EE}}=\frac{4 \kappa H}{\delta}+\mathcal{O}\left(\delta^{0}\right), \quad 1 / 2<m<1 .
$$

\footnotetext{
${ }^{23} \mathrm{~A}$ similar approach is proposed in [79].
} 

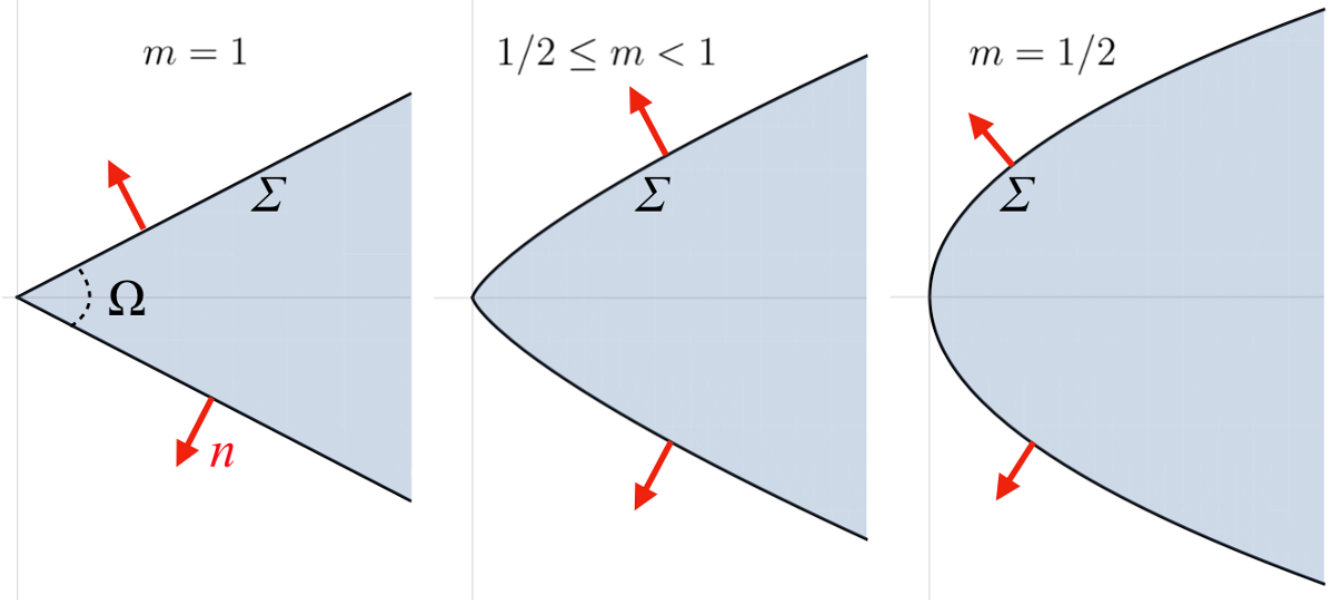

Figure 6. We plot entangling regions on a time slice of a three-dimensional CFT bounded by the curves $y(x)= \pm \lambda x^{m}(x \geq 0)$ with $m=1$ (left), $1 / 2 \leq m<1$ (middle) and $m=1 / 2$. The usual logarithmic universal term in the entanglement entropy $\sim a(\Omega) \log (H / \delta)$ characteristic of the straight corner $(m=1)$ is no longer present for $m<1$, even though the entangling surface is still singular at the tip for $1 / 2 \leq m<1$.

in that case. This means that, even though a geometric singularity is present in the entangling region for $1 / 2<m<1$, no UV divergence appears in the entanglement entropy besides the usual area-law, and the universal contribution is a constant term, just like in the case of smooth regions. Note that no new UV divergence is expected for $m=1 / 2$ since the entangling curve $\Sigma$ is non-singular, being a parabola.

The situation changes for $m \geq 1$, corresponding to sharper corners. In order to study those, let us modify the setup slightly and consider the case of corners formed by the intersection of the curves $y(x)=0$ (i.e. the $x$ axis) and $y(x)=\lambda x^{m},(x>0)$ - see figure 7 . This simplifies computations.

The result for the entanglement entropy is now given by $S_{\mathrm{EE}}=s_{1}(m, \lambda)+s_{1}(1,0)+$ $s_{2}(1,0 ; m, \lambda)$. Again using eq. (4.12), one can show that

$$
\begin{aligned}
s_{1}(m>1, \lambda) & =\frac{2 \kappa H}{\delta}-\kappa \log \left(\frac{H}{\delta}\right)+\mathcal{O}\left(\delta^{0}\right), \\
s_{2}(1,0 ; m>1, \lambda) & =-\frac{2 \kappa \pi c_{m}}{\lambda^{\frac{1}{m}} \delta^{1-\frac{1}{m}}}+2 \kappa \log \left(\frac{H}{\delta}\right)+\mathcal{O}\left(\delta^{0}\right),
\end{aligned}
$$

where the $c_{m}$ are positive non-universal dimensionless constants. Observe that the coefficient of the logarithmic term in $s_{1}(m>1, \lambda)$ differs from the one found for $1 / 2 \leq m<1$ in eq. (4.16). Combining these expressions with eq. (4.13) we are left with

$$
S_{\mathrm{EE}}=\frac{4 \kappa H}{\delta}-\frac{2 \kappa \pi c_{m}}{\lambda^{\frac{1}{m}} \delta^{1-\frac{1}{m}}}+\mathcal{O}\left(\delta^{0}\right) .
$$

Hence, the logarithmic divergence conspires to disappear, and we are left instead with a new non-universal divergence $\sim 1 /\left(\delta^{1-\frac{1}{m}}\right)$ which has precisely the same form as the one found in our mutual information computations of the previous subsection. 


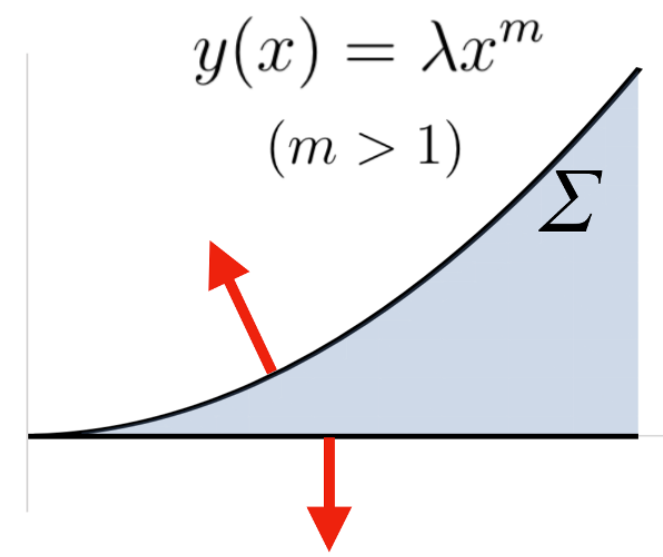

Figure 7. We plot an entangling region on a time slice of a three-dimensional CFT bounded by the curves $y=0$ and $y(x)=\lambda x^{m}(x \geq 0)$ with $m>1$. No logarithmic term is present in the entanglement entropy. Instead, a new non-universal divergence $\sim 1 / \delta^{\left(1-\frac{1}{m}\right)}$ appears.

As we have mentioned, we expect the results obtained in this section to be (qualitatively) valid for general CFTs. In this sense, note that whenever the entangling region is very sharp - in the sense that the entangling surfaces $\Sigma_{1}, \Sigma_{2}$ with $\Sigma=\Sigma_{1} \cup \Sigma_{2}$ are very close to each other along some direction (say, along $y$ ) — one could imagine cutting the entangling region in small thin rectangles which would contribute to the total mutual information in an additive way, effectively making the mutual information become extensive - see also comments at the end of section 4.1. Heuristically, this would suggest a contribution of the form

$$
S_{\mathrm{EE}} \sim \int_{y^{-1}(\delta)}^{L} \frac{d x}{y(x)}
$$

which would be the sum of the contributions coming from the small rectangles of width $d x$ and height $y(x)$, and where the short-distance UV cutoff needs to be imposed along the direction in which the two entangling surfaces are very close to each other ( $y$ in this case). This precisely yields the kind of contributions obtained from the EMI model, e.g., for $y(x)=\lambda x^{m}$,

$$
S_{\mathrm{EE}} \sim \int_{(\delta / \lambda) \frac{1}{m}}^{L} \frac{d x}{\lambda x^{m}} \sim \frac{1}{\lambda^{\frac{1}{m}} \delta^{1-\frac{1}{m}}}
$$

which is the same kind of divergence we obtained in eq. (4.21). If we consider an even sharper corner like $y(x)=e^{-\frac{1}{x}}$, eq. (4.22) produces a divergence of the form

$$
S_{\mathrm{EE}} \sim \frac{1}{\delta \log ^{2} \delta}
$$

which is more divergent than the one produced by any of the power-law corners in eq. (4.23). The sharper the corner, the closer this contribution gets to the area-law divergence, without ever reaching it. 


\section{Conclusion}

In this paper we have presented several new results involving the structure and nature of universal and divergent terms in the von Neumann and Rényi entanglement entropies arising from the presence of geometric singularities on the entangling surface. An in-depth summary of our findings can be found in section 1.1. Let us close the paper with some final words regarding a few possible directions.

One of our main motivations was trying to gain a better understanding on the nature of the trihedral universal coefficient $v_{n}\left(\theta_{1}, \theta_{2}, \theta_{3}\right)$, previously studied using lattice techniques in [58-61], and analytically in the nearly smooth limit [62]. Our results indicate that an analytic computation of this coefficient at general angles for free fields would be equivalent to evaluating partition functions on a $\mathbb{S}^{3}$ with multiplicative boundary conditions on a twodimensional spherical triangle. The methods required to perform this computation have yet to be developed. An analogous computation for the corner region in one dimension less - $\mathbb{S}^{2}$ partition function with boundary conditions on an arc - gives the result in terms of non-linear ordinary differential equations as a function of the angle. Analogously to this case, it is expected that the trihedral coefficient would be given by the solution to some system of non-linear partial differential equations on the two angular variables describing the trihedral angle. Just like in the case of the $2+1$ corner, we do not see that a particular simplification should occur for any particular value of the angles (except in the almostsmooth limit considered in [62]), so the full calculation would need to be addressed, even if one wanted to focus only on the straight-angles case corresponding to $v_{n}(\pi / 2, \pi / 2, \pi / 2)$.

On a different front, let us mention that a behavior similar to the one presented in section 4 regarding the interplay between singularities in the entangling region and the structure of divergences of the Rényi and entanglement entropies is expected to occur for higher-dimensional CFTs - e.g., for smoothed and sharpened (hyper)conical regions. This could be again tested using the EMI model or, in the four-dimensional case, Solodukhin's formula eq. (2.60).

Finally, regarding the question addressed in appendix B, it should not be difficult to find out whether circular cones globally maximize the entanglement entropy within the family of elliptic cones or, more generally, cones generated by moving a straight line emanating from a vertex in a closed orbit.

\section{Acknowledgments}

We thank Marco Baggio, Clément Berthiere, Joan Camps, Rob Myers and Carlos S. Shahbazi for useful discussions. The work of $\mathrm{PB}$ and $\mathrm{HC}$ was supported by the Simons foundation through the It From Qubit Simons collaboration. HC also acknowledges support from CONICET, CNEA and Universidad Nacional de Cuyo, Argentina. WWK was funded by a Discovery Grant from NSERC, a Canada Research Chair, and a "Établissement de nouveaux chercheurs et de nouvelles chercheuses universitaires" grant from FRQNT. 


\section{A Cone entanglement in the Extensive Mutual Information model}

In this appendix we compute the entanglement entropy for a conical entangling surface in $d=4$ in the EMI model. As we have mentioned, we expect the corresponding universal $\log ^{2} \delta$ term to be controlled by a theory-independent function of the opening angle $4 a_{n}^{(4)}(\Omega) / f_{b}(n)=\cos ^{2} \Omega / \sin \Omega$. The EMI should not be an exception, and here we explicitly verify that this is indeed the case.

As explained in section 2.2.1, the general expression for the entanglement entropy in the EMI model is given by eq. (2.31). Let us parametrize the cone surface in cylindrical coordinates by $z=\rho / \tan \Omega, t=0$. Naturally, the line element of flat space in these coordinates reads: $d s^{2}=-d t^{2}+d z^{2}+d \rho^{2}+\rho^{2} d \phi^{2}$. The induced metric on the cone surface is given by $d s_{h}^{2}=d \rho^{2} / \sin ^{2} \Omega+\rho^{2} d \phi$, so $d^{2} \mathbf{r}=[\rho / \sin \Omega] d \rho d \phi$ and analogously for $\mathbf{r}^{\prime}$. Given the symmetry of the problem, we can just set $\phi^{\prime}=0$ everywhere and multiply the remainder integrals by an overall $2 \pi$. The unit normal vector to the cone surface is given $\vec{n}=\vec{u}_{\rho} \cos \Omega-\vec{u}_{z} \sin \Omega$, where $\vec{u}_{\rho}=\cos \phi \vec{u}_{x}+\sin \phi \vec{u}_{y}$. Using this, it is straightforward to find

$$
\mathbf{n}(\mathbf{r}) \cdot \mathbf{n}\left(\mathbf{r}^{\prime}\right)=\cos ^{2} \Omega \cos \phi+\sin ^{2} \Omega .
$$

Similarly, we find $\left|\mathbf{r}-\mathbf{r}^{\prime}\right|^{4}=\left[\rho^{2}+\rho^{\prime 2}-2 \rho \rho^{\prime} \cos \phi+\left(\rho-\rho^{\prime}\right)^{2} / \tan ^{2} \Omega\right]^{2}$. Then, after some trivial manipulations, using eq. (4.1) we are left with the integrals

$$
S_{\mathrm{EE}}=\frac{2 \pi \kappa}{\sin ^{2} \Omega} \int \rho d \rho \int \rho^{\prime} d \rho^{\prime} \int_{0}^{2 \pi} d \phi \frac{\left[\cos ^{2} \Omega \cos \phi+\sin ^{2} \Omega\right]}{[a-b \cos \phi]^{2}},
$$

where $a \equiv \rho^{2}+\rho^{\prime 2}+\left(\rho-\rho^{\prime}\right)^{2} / \tan ^{2} \Omega, b \equiv 2 \rho \rho^{\prime}$, and where we regulate the radial integrals as follows

$$
\int d \rho \equiv \int_{\delta}^{H} d \rho, \quad \int d \rho^{\prime} \equiv\left[\int_{0}^{\rho-\delta} d \rho^{\prime}+\int_{\rho+\delta}^{\infty} d \rho^{\prime}\right]
$$

The angular integrals can be performed using

$$
\int_{0}^{2 \pi} \frac{d \phi}{(a-b \cos \phi)^{2}}=\frac{2 \pi a}{\left(a^{2}-b^{2}\right)^{3 / 2}}, \quad \int_{0}^{2 \pi} \frac{d \phi \cos \phi}{(a-b \cos \phi)^{2}}=\frac{2 \pi b}{\left(a^{2}-b^{2}\right)^{3 / 2}} .
$$

We are left with

$$
S_{\mathrm{EE}}=\frac{4 \pi^{2} \kappa}{\sin ^{2} \Omega}\left[\cos ^{2} \Omega s_{1}+\sin ^{2} \Omega s_{2}\right],
$$

where

$$
s_{1}=\iint \frac{b \rho \rho^{\prime}}{\left(a^{2}-b^{2}\right)^{3 / 2}} d \rho d \rho^{\prime}, \quad s_{2}=\iint \frac{a \rho \rho^{\prime}}{\left(a^{2}-b^{2}\right)^{3 / 2}} d \rho d \rho^{\prime},
$$

with the help of Mathematica we can perform the radial integrals, and the results read

$$
s_{1}=\frac{1}{64} \sin \Omega[\cos (2 \Omega)-7] \log ^{2}\left(\frac{H}{\delta}\right), \quad s_{2}=\frac{1}{32} \cos ^{2} \Omega \sin \Omega \log ^{2}\left(\frac{H}{\delta}\right)
$$

up to nonuniversal contributions. Putting both pieces together, we are left with

$$
S_{\mathrm{EE}}=-\frac{3 \pi^{2} \kappa}{8} \cdot \frac{\cos ^{2} \Omega}{\sin \Omega} \log ^{2}\left(\frac{H}{\delta}\right)
$$


which takes the expected form. We can now write the coefficient $\kappa$ in terms of the traceanomaly charge $c_{\mathrm{EMI}}$, e.g., using the result for the entanglement entropy of a cylinder in the EMI model and comparing it with the general one following from Solodukhin's formula eq. (2.42). The result reads

Using this, we find

$$
c_{\mathrm{EMI}}=\frac{3 \kappa}{2 \pi^{2}} .
$$

$$
S_{\mathrm{EE}}=-\frac{c}{4} \cdot \frac{\cos ^{2} \Omega}{\sin \Omega} \log ^{2}\left(\frac{H}{\delta}\right),
$$

which is the exact result valid for general CFTs - i.e. it already contains the "famous" $1 / 2$ factor that is missing in the calculation using Solodukhin's formula.

\section{B Which cone maximizes the Rényi entropy?}

In section 2 , we found how the universal cone formula $a_{n}^{(4)}(\Omega)=f_{b}(n) \frac{\cos ^{2} \Omega}{4 \sin \Omega}$ is modified when the cross-sections of the cones become ellipses rather than circles. We showed that the full result for the Rényi entropy reads in that case

$$
S_{n}=b_{2} \frac{H^{2}}{\delta^{2}}-\frac{f_{b}(n)}{4} \gamma\left(e^{\prime}, \theta_{0}\right) \log ^{2}\left(\frac{R}{\delta}\right)
$$

where $\gamma\left(e^{\prime}, \theta_{0}\right)$ was given in eq. (2.64) as a function of the ellipses second-eccentricity $e^{\prime}$ and the semi-opening angle $\theta_{0}$.

A natural question one is led to ask is which cone extremizes the Rényi entropy within the family of elliptic cones. In the case of compact smooth surfaces, this question was addressed in [80], where it was shown that the round sphere $\mathbb{S}^{2}$ is the one which maximizes it both within the family of genus- 0 surfaces and for general-genus surfaces. ${ }^{24}$ A meaningful comparison of this kind can be performed by fixing the lateral area of the cones, so that the area-law terms cancel each other when the difference is considered. The lateral area of the elliptic cones is given by

$$
A=2 \int_{0}^{\pi / 2} \frac{R^{2} \sin \theta_{0} \sqrt{1+e^{\prime 2} \cos ^{2} \phi}}{\sqrt{1+e^{\prime 2} \sin ^{2} \theta_{0} \cos ^{2} \phi}} d \phi,
$$

which depends on whether we cut it off at a fixed $R$ or at some height $z=z_{0}$. In the second case, we need to replace $R$ by $R\left(z_{0}, \phi\right)=z_{0} \sqrt{1+e^{\prime 2} \sin ^{2} \theta_{0}} /\left(\cos \theta_{0} \sqrt{1-e^{\prime 2} \sin ^{2} \theta_{0} \cos ^{2} \phi}\right)$. Then, the result can be expressed in terms of the complete elliptic integral of the second kind $E[x]$ as $^{25}$

$$
A_{\left(z_{0}\right)}=\frac{2 z_{0}^{2} \sin \theta_{0}}{\cos ^{2} \theta_{0}} \sqrt{1+e^{\prime 2} \sin ^{2} \theta_{0}} E\left[\frac{-e^{\prime 2} \cos ^{2} \theta_{0}}{1+e^{\prime 2} \sin ^{2} \theta_{0}}\right] .
$$

\footnotetext{
${ }^{24}$ For fixed genus, the surfaces maximizing the Rényi entropy turn out to correspond to the so-called Lawson surfaces, namely, surfaces which can be minimally embedded in $\mathbb{S}^{3}$ - see [80] for details.

${ }^{25}$ This can be written in terms of the ellipses semi-axes as

$$
A_{\left(z_{0}\right)}=2 a \sqrt{z_{0}^{2}+b^{2}} E\left[\frac{1-b^{2} / a^{2}}{1+b^{2} / z_{0}^{2}}\right],
$$

which is a relatively well-known result. Note that different sources define $E[z]$ in a slightly different way: $E[z] \equiv \int_{0}^{\pi / 2} d t \sqrt{1-z \sin ^{2} t}$ vs $E[z] \equiv \int_{0}^{\pi / 2} d t \sqrt{1-z^{2} \sin ^{2} t}$. Here we use the first definition, which is the one implemented in Mathematica.
} 
Conditions $A_{(R)}=$ constant and $A_{\left(z_{0}\right)}=$ constant cannot be easily converted into explicit relations between $\theta_{0}$ and $e^{\prime}$. They can nonetheless be implemented for small $e^{\prime}$. In the first case, one finds

$$
\left.\gamma\left(e^{\prime}\right)\right|_{A_{(R)}}=\frac{1-\bar{A}_{(R)}^{2}}{\bar{A}_{(R)}}\left[1+\frac{3\left(3+\bar{A}_{(R)}^{2}\right)}{32} e^{\prime 4}+\mathcal{O}\left(e^{\prime 6}\right)\right],
$$

where we defined $\bar{A}_{(R)} \equiv A_{(R)} /\left(\pi R^{2}\right)$, which satisfies $\bar{A}_{(R)} \leq 1$ for $e^{\prime}<1$. Interestingly, the quadratic term in $e^{\prime}$ appearing in eq. (2.68) disappears when we keep $A_{(R)}$ fixed. As a consequence, circular cones are minima of $\gamma$ when compared to other cones of the same lateral area and cutoff at some radial distance $R$ but different elliptic cross-sections. Since $\gamma$ contributes with a negative sign to the Rényi entropy, circular cones are maxima of the Rényi entropy within this family.

If we impose $A_{\left(z_{0}\right)}=$ constant instead, we obtain

$$
\left.\gamma\left(e^{\prime}\right)\right|_{A_{\left(z_{0}\right)}}=\frac{1}{\bar{A}_{\left(z_{0}\right)}}\left[1+\frac{1+\left(6 \bar{A}_{\left(z_{0}\right)}^{2}-\sqrt{1+4 \bar{A}_{\left(z_{0}\right)}^{2}}\right)\left(1+4 \bar{A}_{\left(z_{0}\right)}^{2}\right)}{64 \bar{A}_{\left(z_{0}\right)}^{4}} e^{\prime 4}+\mathcal{O}\left(e^{\prime 6}\right)\right]
$$

where we now defined $\bar{A}_{\left(z_{0}\right)} \equiv A_{\left(z_{0}\right)} /\left(\pi z_{0}^{2}\right)$. Once again, the quadratic term in eq. (2.68) conspires to disappear, and the quartic coefficient is always positive. Hence, circular cones are also local maximizers of the Rényi entropy within the class of elliptic cones cutoff at a fixed height $z_{0}$. It would be interesting to verify whether this holds globally within this family.

More generally, it would be interesting to find out if circular cones are maximizers of the Rényi entropy for "straight" cones, i.e. those generated by moving a straight line in a closed orbit.

\section{Hyperconical entanglement in even dimensions}

In this appendix we argue that the universal contribution to the Rényi entropy of right circular (hyper)cones in general even-dimensional CFTs is given by the simple formula eq. (2.76). Namely, the universal contribution to the Rényi entropy for a (hyper)conical region, which is quadratically logarithmic in the cutoff, is such that the function of the opening angle consists of the four-dimensional result, $\cos ^{2} \Omega / \sin \Omega$, times a linear combination of the form: $\gamma_{0, n}^{(d)}+\gamma_{1, n}^{(d)} \cos (2 \Omega)+\gamma_{2, n}^{(d)} \cos (4 \Omega)+\cdots+\gamma_{(d-4) / 2, n}^{(d)} \cos ((d-4) \Omega)$. The only theory-dependent input appears through coefficients $\gamma_{j, n}^{(d)}$ which, in the entanglement entropy case, are linear combinations of the trace-anomaly charges characterizing the corresponding CFT - e.g., for four-dimensional theories, $\gamma_{0, n}^{(4)}=f_{b}(n) / 4$, and for six-dimensional theories, $\gamma_{0,1}^{(4)}$ and $\gamma_{1,1}^{(4)}$ can be obtained from the results in $[9,10,52]$.

The universal contribution to the Rényi entropy of smooth entangling regions on evendimensional CFTs is logarithmically divergent, the universal coefficient given by sums of local integrals on the corresponding entangling surfaces $\Sigma$, weighted by linear combinations 
of the trace-anomaly coefficients $[7-10,81]$. One of the terms is always controlled by the "a-type" charge (or its Rényi generalization), whose corresponding local integral is proportional to the Euler characteristic of $\Sigma$ - namely, it corresponds to the $(d-2)$ dimensional Euler density integrated over $\Sigma$. On the (hyper)conincal surfaces, all these intrinsic-curvature terms vanish, except at the tip, where all curvature is concentrated, in a way such that if we close the cone at some finite distance, the contribution from this term equals the corresponding topological invariant. Since we work with semi-infinite cones, we shall not be concerned by this contribution. In addition, there are a number of contributions which involve integrals of various combinations of the extrinsic curvature of $\Sigma$. These must be invariant under local diffeomorphisms on $\Sigma$, and their possible linear combinations must be chosen such that conformal invariance is respected. Attending to the first requirement, we can divide the different terms into two categories. The first class of terms takes the generic form

$$
k^{n}\left[\operatorname{tr} k^{m}\right]^{s},
$$

where we use the notation $\left(\operatorname{tr} k^{m}\right)^{s} \equiv\left(k_{a_{1}}{ }^{a_{2}} k_{a_{2}}{ }^{a_{3}} \ldots k_{a_{m}}{ }^{a_{1}}\right)^{s}$, and where $n, m$ and $s$ are non-negative integers constrained to satisfy $(n+m s)=(d-2), m \geq 2$. Hence, for example, in $d=4$ all possible terms reduce to two: $k^{2}$ and $\operatorname{tr} k^{2}$. In $d=6$ there are more options, namely

$$
k^{4}, \quad k^{2} \operatorname{tr} k^{2}, \quad k \operatorname{tr} k^{3}, \quad \operatorname{tr} k^{4}, \quad\left(\operatorname{tr} k^{2}\right)^{2} .
$$

In $d=8$, in turn, we have eight possibilities,

$$
k^{6}, \quad k^{4} \operatorname{tr} k^{2}, \quad k^{3} \operatorname{tr} k^{3}, \quad k^{2} \operatorname{tr} k^{4}, \quad k \operatorname{tr} k^{5}, \quad \operatorname{tr} k^{6}, \quad k^{2}\left(\operatorname{tr} k^{2}\right)^{2}, \quad\left(\operatorname{tr} k^{2}\right)^{3} .
$$

And similarly in higher dimensions.

The second class of terms - which in fact includes the first as a particular case involves covariant derivatives of the extrinsic curvature. The most general form of one of those terms consists of some contraction of

$$
\underbrace{k_{. .} \cdots k_{.}}_{\alpha_{1}} \underbrace{\left(\nabla . k_{. .}\right) \cdots\left(\nabla . k_{. .}\right)}_{\alpha_{2}} \underbrace{\left(\nabla . \nabla . k_{. .}\right) \cdots\left(\nabla \cdot \nabla \cdot k_{. .}\right)}_{\alpha_{3}} \cdots \underbrace{\left(\nabla . \nabla . \cdots \nabla . k_{. .}\right) \cdots\left(\nabla . \nabla \cdot \cdots \nabla . k_{. .}\right)}_{\alpha_{r}},
$$

with $\frac{1}{2} \sum_{i}^{r}(i+1) \alpha_{i}$ inverse metrics. Observe that in eq. (C.4) we have $\alpha_{\text {tot }} \equiv \sum_{j}^{r} \alpha_{j}$ terms in total, with $\alpha_{j}$ of them involving $j-1$ covariant derivatives of the extrinsic curvature. The total number of terms is always an even number, $\alpha_{\text {tot }}=$ even, and it is bounded below and above by $2 \leq \alpha_{\text {tot }} \leq d-2$. Similarly, the $\alpha_{j}$ are constrained to satisfy

$$
\sum_{i=1}^{r} i \cdot \alpha_{i}=d-2 .
$$

This captures the intuition that, for dimensionality purposes, one covariant derivative counts as one extrinsic curvature. For example, in $d=4$, this condition reads $\alpha_{1}+2 \alpha_{2}=2$, which means that, in principle, we could have contractions of two extrinsic curvatures $\left(\alpha_{1}=2\right)$ or, alternatively, one contraction involving $\nabla_{a} k_{b c},\left(\alpha_{2}=1\right)$, but since the total 
number of terms must be even, the latter case is discarded, and no terms involving covariant derivatives are allowed.

The particular combination of terms appearing in the entanglement/Rényi entropy expressions and their respective weights as functions of the coefficients appearing in the trace-anomaly expressions have been worked out explicitly in $d=4$ and $d=6[7,9,10]$. As we show now, in the case of entangling surfaces consisting in (hyper)cones, a general pattern exists regarding the functional dependence of $a_{n}^{(d)}(\Omega)$.

The metric of $d$-dimensional Minkowski space in hyperspherical coordinates can be written as

$$
d s^{2}=-d t^{2}+d r^{2}+r^{2}\left[d \theta^{2}+\sin ^{2} \theta\left(d \theta_{1}^{2}+\sin ^{2} \theta_{1}\left(d \theta_{2}^{2}+\sin ^{2} \theta_{2}^{2}\left(d \theta_{3}^{2}+\sin ^{2} \theta_{3}(\ldots],\right.\right.\right.\right.
$$

where the coordinate ranges are: $\theta, \theta_{1}, \theta_{2}, \ldots, \theta_{(d-4)} \in[0, \pi]$ and $\theta_{(d-3)} \in[0,2 \pi)$, which is the usual $\phi$ coordinate in $d=4$. Hypercones are parametrized by equations: $t=0$ and $\theta=\Omega$. It is then straightforward to obtain the corresponding induced metric, and its determinant,

$$
\begin{aligned}
& d s_{h}^{2}=d r^{2}+r^{2} \sin ^{2} \Omega\left[d \theta_{1}^{2}+\sin ^{2} \theta_{1}\left(d \theta_{2}^{2}+\ldots\right],\right. \\
& \sqrt{h}=(r \sin \Omega)^{(d-3)} \prod_{j=1}^{d-4} \sin ^{(d-3-j)} \theta_{j} .
\end{aligned}
$$

The only non-vanishing components of the extrinsic curvature associated to the normal vector $n=\frac{1}{\sqrt{g_{\theta \theta}}} \partial_{\theta} \operatorname{read}^{26}$

$$
k_{\theta_{1} \theta_{1}}=r \cos \Omega \sin \Omega, \quad k_{\theta_{i} \theta_{i}}=k_{\theta_{1} \theta_{1}} \prod_{j=1}^{i} \sin ^{2} \theta_{j}, \quad i=2, \ldots, d-3 .
$$

Now, using the fact that the only non-vanishing components of $h^{a b}$ are given by

$$
h^{\theta_{1} \theta_{1}}=\frac{1}{r^{2} \sin ^{2} \Omega}, \quad h^{\theta_{i} \theta_{i}}=h^{\theta_{1} \theta_{1}} \prod_{j=1}^{i} \frac{1}{\sin ^{2} \theta_{j}}, \quad i=2, \ldots, d-3,
$$

it is easy to show that

$$
\begin{aligned}
\int_{\Sigma} d^{d-2} y \sqrt{h} k^{n}\left[\operatorname{tr} k^{m}\right]^{s} & =\frac{\cos ^{(d-2)} \Omega}{\sin \Omega}(d-3)^{(n+s)} \int \frac{d r}{r} \int_{0}^{\pi} 2 \pi \prod_{j=1}^{d-4} \sin ^{(d-3-j)} \theta_{j} d \theta_{j}, \\
& =\frac{2 \pi^{\frac{d-2}{2}}(d-3)^{(n+s)}}{\Gamma\left[\frac{d-2}{2}\right]} \frac{\cos ^{(d-2)} \Omega}{\sin \Omega} \int \frac{d r}{r}
\end{aligned}
$$

Here, we observe the appearance of a $\int d r / r$ factor which, independent of the dimension, combines in each case with the overall $\log \delta$ to produce a $\log ^{2} \delta$ divergence in exactly the same way as for the $d=4$ case discussed in section 2 - and again there will be a missing

\footnotetext{
${ }^{26}$ The extrinsic curvature associated to the other normal, $n^{(1)}=\partial_{t}$, is trivial in this case and we just ignore it here.
} 
$1 / 2$ factor. We also observe that the dependence on the cone opening angle is modified with respect to the $d=4$ case by the appearance of a different power for $\cos \Omega$. We can alternatively think of this as producing additional terms proportional to $\cos (2 j \Omega)$ with $j=1, \ldots,(d-4) / 2$ multiplying the overall $d=4$ result. Indeed, using the identity

$$
\cos ^{(d-2)} \Omega=\frac{\cos ^{2} \Omega}{2^{d-4}}\left[\left(\begin{array}{c}
d-4 \\
\frac{d-4}{2}
\end{array}\right)+2 \sum_{j=1}^{\frac{d-4}{2}}\left(\begin{array}{c}
d-4 \\
\frac{d-4-2 j}{2}
\end{array}\right) \cos (2 j \Omega)\right],
$$

we find

$$
\begin{aligned}
\int_{\Sigma} d^{d-2} y \sqrt{h} k^{n}\left[\operatorname{tr} k^{m}\right]^{s}= & \frac{\pi^{\frac{d-2}{2}}(d-3)^{(n+s)}}{2^{d-5} \Gamma\left[\frac{d-2}{2}\right]} \frac{\cos ^{2} \Omega}{\sin \Omega} \\
& \times\left[\left(\begin{array}{c}
d-4 \\
\frac{d-4}{2}
\end{array}\right)+2 \sum_{j=1}^{\frac{d-4}{2}}\left(\begin{array}{c}
d-4 \\
\frac{d-4-2 j}{2}
\end{array}\right) \cos (2 j \Omega)\right] \int \frac{d r}{r},
\end{aligned}
$$

which takes the form suggested in eq. (2.76).

We turn now to the second type of contributions, i.e. those of the general form eq. (C.4). We cannot be as explicit in this case, but the general pattern can be also understood. As we explained earlier, integrands for this kind of terms will take the generic form

$$
\int_{\Sigma} d^{d-2} y \sqrt{h} \underbrace{k \ldots \cdots}_{a_{1}} \underbrace{\left(\nabla . k_{. .}\right) \cdots}_{a_{2}} \underbrace{\left(\nabla . \nabla . k_{.}\right) \cdots}_{a_{3}} \cdots \underbrace{\left(\nabla . \nabla . \cdots \nabla . k_{. .}\right) \cdots}_{a_{r}} \underbrace{h^{\cdots} \cdots h^{*}}_{\frac{1}{2} \sum_{i}^{r}(i+1) a_{i}} .
$$

Using eq. (C.9) and eq. (C.10), we find that in the case of the hypercones this generically reduces to

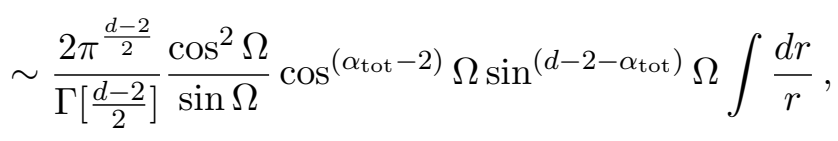

up to a constant that depends on the dimension and the specific index structure in eq. (C.14).

Now $\sin ^{\left(d-2-\alpha_{\text {tot }}\right)} \Omega$ can be expanded in even powers of $\cos \Omega$ so that

$$
\cos ^{\left(\alpha_{\mathrm{tot}}-2\right)} \Omega \sin ^{\left(d-2-\alpha_{\mathrm{tot}}\right)} \Omega=\sum_{k=0}^{\frac{d-2-\alpha_{\mathrm{tot}}}{2}}\left(\begin{array}{c}
\frac{d-2-\alpha_{\mathrm{tot}}}{2} \\
k
\end{array}\right)(-1)^{k} \cos ^{\left(2 k+\alpha_{\mathrm{tot}}-2\right)} \Omega .
$$

Finally, expanding $\cos ^{\left(2 k+\alpha_{\text {tot }}-2\right)} \Omega$ as in eq. (C.12) we are left with a linear combination of terms of the form $\cos (2 j \Omega)$. It is straightforward to show that the condition $2 \leq \alpha_{\text {tot }} \leq d-2$ constrains the possible values of $2 j$ to $2 j=0,2, \cdots, d-4$, in agreement with the conjectural relation eq. (2.76), which therefore holds in general. In passing, we note that we have actually verified explicitly that eq. (2.76) holds for the holographic entanglement entropy of CFTs dual to Einstein gravity in $d=4,6,8,10,12$. In that case, the bulk action is given 
by eq. (3.21). Closely following the calculations in [25] for the $d=4,6$ cases, we obtain

$$
\begin{aligned}
a^{(4)}(\Omega)= & \frac{\cos ^{2} \Omega}{\sin \Omega}\left(\frac{\pi L^{3}}{32 G}\right) \\
a^{(6)}(\Omega)= & \frac{\cos ^{2} \Omega}{\sin \Omega}\left(\frac{9 \pi^{2} L^{5}}{16384 G}\right)[31-\cos (2 \Omega)] \\
a^{(8)}(\Omega)= & \frac{\cos ^{2} \Omega}{\sin \Omega}\left(\frac{25 \pi^{3} L^{7}}{95551488 G}\right)[22353-964 \cos (2 \Omega)+11 \cos (4 \Omega)], \\
a^{(10)}(\Omega)= & \frac{\cos ^{2} \Omega}{\sin \Omega}\left(\frac{49 \pi^{4} L^{9}}{14843406974976 G}\right)[449662142-21823587 \cos (2 \Omega) \\
& +370802 \cos (4 \Omega)-2669 \cos (6 \Omega)] \\
a^{(12)}(\Omega)= & \frac{\cos ^{2} \Omega}{\sin \Omega}\left(\frac{27 \pi^{5} L^{11}}{83886080000000000 G}\right)[930830869835 \\
& -48180346664 \cos (2 \Omega)+977555068 \cos (4 \Omega)-11174552 \cos (6 \Omega) \\
& +53881 \cos (8 \Omega)]
\end{aligned}
$$

which indeed respect the aforementioned angular dependence.

Observe that, on general grounds, in the sharp and almost-smooth limits, the functions $a_{n}^{(d)}(\Omega)$ behave similarly to the $d=4$ case (or the $d=3$ corner), namely

$$
\begin{aligned}
& a_{n}^{(d)}(\Omega) \stackrel{\Omega \rightarrow 0}{=} \sum_{j=0}^{\frac{d-4}{2}}\left[\frac{1}{\Omega}-\left[\frac{5}{6}+2 j^{2}\right] \Omega+\mathcal{O}\left(\Omega^{2}\right)\right] \gamma_{j, n}^{(d)} \\
& a_{n}^{(d)}(\Omega) \stackrel{\Omega \rightarrow \pi / 2}{=} \sum_{j=0}^{\frac{d-4}{2}}\left[\left(\Omega-\frac{\pi}{2}\right)^{2}+\left[\frac{1}{6}-2 j^{2}\right]\left(\Omega-\frac{\pi}{2}\right)^{4}+\mathcal{O}\left(\Omega-\frac{\pi}{2}\right)^{6}\right](-1)^{j} \gamma_{j, n}^{(d)} .
\end{aligned}
$$

Open Access. This article is distributed under the terms of the Creative Commons Attribution License (CC-BY 4.0), which permits any use, distribution and reproduction in any medium, provided the original author(s) and source are credited.

\section{References}

[1] T. Nishioka, Entanglement entropy: holography and renormalization group, Rev. Mod. Phys. 90 (2018) 035007 [arXiv: 1801.10352] [INSPIRE].

[2] E. Witten, APS medal for exceptional achievement in research: invited article on entanglement properties of quantum field theory, Rev. Mod. Phys. 90 (2018) 045003 [arXiv: 1803.04993] [INSPIRE].

[3] T. Grover, A.M. Turner and A. Vishwanath, Entanglement entropy of gapped phases and topological order in three dimensions, Phys. Rev. B 84 (2011) 195120 [arXiv:1108.4038] [INSPIRE].

[4] H. Liu and M. Mezei, A refinement of entanglement entropy and the number of degrees of freedom, JHEP 04 (2013) 162 [arXiv:1202.2070] [INSPIRE]. 
[5] P. Calabrese and J.L. Cardy, Entanglement entropy and quantum field theory, J. Stat. Mech. 0406 (2004) P06002 [hep-th/0405152] [INSPIRE].

[6] P. Calabrese and J. Cardy, Entanglement entropy and conformal field theory, J. Phys. A 42 (2009) 504005 [arXiv:0905.4013] [inSPIRE].

[7] S.N. Solodukhin, Entanglement entropy, conformal invariance and extrinsic geometry, Phys. Lett. B 665 (2008) 305 [arXiv: 0802.3117] [INSPIRE].

[8] D.V. Fursaev, Entanglement Rényi entropies in conformal field theories and holography, JHEP 05 (2012) 080 [arXiv: 1201.1702] [INSPIRE].

[9] B.R. Safdi, Exact and numerical results on entanglement entropy in $(5+1)$-dimensional CFT, JHEP 12 (2012) 005 [arXiv: 1206.5025] [INSPIRE].

[10] R.-X. Miao, Universal terms of entanglement entropy for 6d CFTs, JHEP 10 (2015) 049 [arXiv: 1503. 05538] [INSPIRE].

[11] H. Casini, Mutual information challenges entropy bounds, Class. Quant. Grav. 24 (2007) 1293 [gr-qc/0609126] [inSPIRE].

[12] H. Casini, M. Huerta, R.C. Myers and A. Yale, Mutual information and the F-theorem, JHEP 10 (2015) 003 [arXiv: 1506.06195] [INSPIRE].

[13] H. Casini, M. Huerta and R.C. Myers, Towards a derivation of holographic entanglement entropy, JHEP 05 (2011) 036 [arXiv:1102.0440] [INSPIRE].

[14] J.S. Dowker, Entanglement entropy for odd spheres, arXiv:1012.1548 [INSPIRE].

[15] H. Casini and M. Huerta, Universal terms for the entanglement entropy in $2+1$ dimensions, Nucl. Phys. B 764 (2007) 183 [hep-th/0606256] [INSPIRE].

[16] H. Casini, M. Huerta and L. Leitao, Entanglement entropy for a Dirac fermion in three dimensions: vertex contribution, Nucl. Phys. B 814 (2009) 594 [arXiv:0811.1968] [INSPIRE].

[17] H. Casini and M. Huerta, Entanglement entropy in free quantum field theory, J. Phys. A 42 (2009) 504007 [arXiv:0905.2562] [INSPIRE].

[18] P. Bueno, R.C. Myers and W. Witczak-Krempa, Universal corner entanglement from twist operators, JHEP 09 (2015) 091 [arXiv: 1507.06997] [INSPIRE].

[19] J.S. Dowker, Note on Rényi vertex contributions and twist operator weights for free scalar fields, arXiv: 1509.00782 [INSPIRE].

[20] J.S. Dowker, Conformal weights of charged Rényi entropy twist operators for free Dirac fields in arbitrary dimensions, arXiv:1510.08378 [INSPIRE].

[21] H. Elvang and M. Hadjiantonis, Exact results for corner contributions to the entanglement entropy and Rényi entropies of free bosons and fermions in 3d, Phys. Lett. B 749 (2015) 383 [arXiv: 1506.06729] [INSPIRE].

[22] C. Berthiere, Boundary-corner entanglement for free bosons, Phys. Rev. B 99 (2019) 165113 [arXiv: 1811.12875] [INSPIRE].

[23] S. Whitsitt, W. Witczak-Krempa and S. Sachdev, Entanglement entropy of the large N Wilson-Fisher conformal field theory, Phys. Rev. B 95 (2017) 045148 [arXiv:1610.06568] [INSPIRE]. 
[24] T. Hirata and T. Takayanagi, AdS/CFT and strong subadditivity of entanglement entropy, JHEP 02 (2007) 042 [hep-th/0608213] [INSPIRE].

[25] R.C. Myers and A. Singh, Entanglement entropy for singular surfaces, JHEP 09 (2012) 013 [arXiv:1206.5225] [INSPIRE].

[26] P. Bueno and R.C. Myers, Corner contributions to holographic entanglement entropy, JHEP 08 (2015) 068 [arXiv:1505.07842] [INSPIRE].

[27] P. Fonda, D. Seminara and E. Tonni, On shape dependence of holographic entanglement entropy in $A d S_{4} / C F T_{3}, J H E P 12$ (2015) 037 [arXiv:1510.03664] [INSPIRE].

[28] R.-X. Miao, A holographic proof of the universality of corner entanglement for CFTs, JHEP 10 (2015) 038 [arXiv:1507.06283] [INSPIRE].

[29] M. Alishahiha, A.F. Astaneh, P. Fonda and F. Omidi, Entanglement entropy for singular surfaces in hyperscaling violating theories, JHEP 09 (2015) 172 [arXiv:1507.05897] [INSPIRE].

[30] D.-W. Pang, Corner contributions to holographic entanglement entropy in non-conformal backgrounds, JHEP 09 (2015) 133 [arXiv:1506.07979] [INSPIRE].

[31] M.R. Mohammadi Mozaffar, A. Mollabashi and F. Omidi, Holographic mutual information for singular surfaces, JHEP 12 (2015) 082 [arXiv: 1511.00244] [INSPIRE].

[32] L. Bianchi et al., Shape dependence of holographic Rényi entropy in general dimensions, JHEP 11 (2016) 180 [arXiv: 1607.07418] [INSPIRE].

[33] D. Seminara, J. Sisti and E. Tonni, Corner contributions to holographic entanglement entropy in $A d S_{4} / B C F T_{3}, J H E P 11$ (2017) 076 [arXiv: 1708.05080] [INSPIRE].

[34] G. Pastras, On the holographic entanglement entropy for non-smooth entangling curves in $A d S_{4}$, Fortsch. Phys. 66 (2018) 1700090 [arXiv:1710.01948] [InSPIRE].

[35] E. Bakhshaei, A. Mollabashi and A. Shirzad, Holographic subregion complexity for singular surfaces, Eur. Phys. J. C 77 (2017) 665 [arXiv:1703.03469] [INSPIRE].

[36] M. Ghasemi and S. Parvizi, Entanglement entropy of singular surfaces under relevant deformations in holography, JHEP 02 (2018) 009 [arXiv: 1709.08169] [INSPIRE].

[37] D. Seminara, J. Sisti and E. Tonni, Holographic entanglement entropy in $A d S_{4} / B C F T_{3}$ and the Willmore functional, JHEP 08 (2018) 164 [arXiv:1805.11551] [INSPIRE].

[38] E. Caceres and M.-L. Xiao, Complexity-action of subregions with corners, JHEP 03 (2019) 062 [arXiv: 1809.09356] [INSPIRE].

[39] M. Ghasemi and S. Parvizi, Relevant perturbation of entanglement entropy of singular surfaces, arXiv: 1902.05058 [INSPIRE].

[40] A.B. Kallin, M.B. Hastings, R.G. Melko and R.R.P. Singh, Anomalies in the entanglement properties of the square-lattice Heisenberg model, Phys. Rev. B 84 (2011) 165134 [arXiv: 1107.2840].

[41] A.B. Kallin et al., Corner contribution to the entanglement entropy of an $O(3)$ quantum critical point in $2+1$ dimensions, J. Stat. Mech. 1406 (2014) P06009 [arXiv:1401.3504] [INSPIRE].

[42] J. Helmes and S. Wessel, Correlations and entanglement in quantum critical bilayer and necklace XY models, Phys. Rev. B 92 (2015) 125120 [arXiv:1411.7773] [INSPIRE]. 
[43] N. Laflorencie, D.J. Luitz and F. Alet, Spin-wave approach for entanglement entropies of the J1-J2 Heisenberg antiferromagnet on the square lattice, Phys. Rev. B 92 (2015) 115126 [arXiv: 1506.03703] [INSPIRE].

[44] J. Helmes et al., Universal corner entanglement of Dirac fermions and gapless bosons from the continuum to the lattice, Phys. Rev. B 94 (2016) 125142 [arXiv:1606.03096] [InSPIRE].

[45] C. De Nobili, A. Coser and E. Tonni, Entanglement negativity in a two dimensional harmonic lattice: area law and corner contributions, J. Stat. Mech. 1608 (2016) 083102 [arXiv: 1604.02609] [INSPIRE].

[46] P. Bueno, R.C. Myers and W. Witczak-Krempa, Universality of corner entanglement in conformal field theories, Phys. Rev. Lett. 115 (2015) 021602 [arXiv:1505.04804] [INSPIRE].

[47] T. Faulkner, R.G. Leigh and O. Parrikar, Shape dependence of entanglement entropy in conformal field theories, JHEP 04 (2016) 088 [arXiv: 1511.05179] [INSPIRE].

[48] P. Bueno and W. Witczak-Krempa, Bounds on corner entanglement in quantum critical states, Phys. Rev. B 93 (2016) 045131 [arXiv:1511.04077] [InSPIRE].

[49] W. Witczak-Krempa, L.E. Hayward Sierens and R.G. Melko, Cornering gapless quantum states via their torus entanglement, Phys. Rev. Lett. 118 (2017) 077202 [arXiv: 1603. 02684] [INSPIRE].

[50] C.-S. Chu and R.-X. Miao, Universality in the shape dependence of holographic Rényi entropy for general higher derivative gravity, JHEP 12 (2016) 036 [arXiv:1608.00328] [INSPIRE].

[51] H. Osborn and A.C. Petkou, Implications of conformal invariance in field theories for general dimensions, Annals Phys. 231 (1994) 311 [hep-th/9307010] [INSPIRE].

[52] P. Bueno and R.C. Myers, Universal entanglement for higher dimensional cones, JHEP 12 (2015) 168 [arXiv: 1508.00587] [INSPIRE].

[53] M. Mezei, Entanglement entropy across a deformed sphere, Phys. Rev. D 91 (2015) 045038 [arXiv: 1411.7011] [INSPIRE].

[54] L. Bianchi, M. Meineri, R.C. Myers and M. Smolkin, Rényi entropy and conformal defects, JHEP 07 (2016) 076 [arXiv: 1511.06713] [INSPIRE].

[55] X. Dong, Shape dependence of holographic Rényi entropy in conformal field theories, Phys. Rev. Lett. 116 (2016) 251602 [arXiv: 1602.08493] [INSPIRE].

[56] S. Balakrishnan, S. Dutta and T. Faulkner, Gravitational dual of the Rényi twist displacement operator, Phys. Rev. D 96 (2017) 046019 [arXiv:1607.06155] [INSPIRE].

[57] I.R. Klebanov, T. Nishioka, S.S. Pufu and B.R. Safdi, On shape dependence and rg flow of entanglement entropy, JHEP 07 (2012) 001 [arXiv:1204.4160] [INSPIRE].

[58] I.A. Kovacs and F. Igoli, Universal logarithmic terms in the entanglement entropy of 2d, $3 d$ and 4 d random transverse-field ising models, Europhys. Lett. 97 (2012) 67009.

[59] T. Devakul and R.R.P. Singh, Entanglement across a cubic interface in $3+1$ dimensions, Phys. Rev. B 90 (2014) 054415 [arXiv:1407.0084].

[60] L.E.H. Sierens et al., Cubic trihedral corner entanglement for a free scalar, Phys. Rev. B 96 (2017) 035117 [arXiv: 1703.03413] [INSPIRE].

[61] G. Bednik et al., Probing trihedral corner entanglement for Dirac fermions, Phys. Rev. B 99 (2019) 155153 [arXiv:1810.02831] [INSPIRE]. 
[62] W. Witczak-Krempa, Entanglement susceptibilities and universal geometric entanglement entropy, Phys. Rev. B 99 (2019) 075138 [arXiv: 1810.07209] [InSPIRE].

[63] M.P. Hertzberg and F. Wilczek, Some calculable contributions to entanglement entropy, Phys. Rev. Lett. 106 (2011) 050404 [arXiv: 1007.0993] [InSPIRE].

[64] H. Casini, F.D. Mazzitelli and E. Testé, Area terms in entanglement entropy, Phys. Rev. D 91 (2015) 104035 [arXiv:1412.6522] [INSPIRE].

[65] M. Huerta, Numerical determination of the entanglement entropy for free fields in the cylinder, Phys. Lett. B 710 (2012) 691 [arXiv:1112.1277] [INSPIRE].

[66] H. Casini, C.D. Fosco and M. Huerta, Entanglement and alpha entropies for a massive Dirac field in two dimensions, J. Stat. Mech. 0507 (2005) P07007 [cond-mat/0505563] [INSPIRE].

[67] H. Casini and M. Huerta, Remarks on the entanglement entropy for disconnected regions, JHEP 03 (2009) 048 [arXiv: 0812.1773] [INSPIRE].

[68] B. Swingle, Mutual information and the structure of entanglement in quantum field theory, arXiv: 1010.4038 [INSPIRE].

[69] R. Helling, H. Leschke and W. Spitzer, A special case of a conjecture by widom with implications to fermionic entanglement entropy, Int. Math. Res. Not. 2011 (2011) 1451 [arXiv: 0906.4946] [INSPIRE].

[70] H. Dorn, On the logarithmic divergent part of entanglement entropy, smooth versus singular regions, Phys. Lett. B 763 (2016) 134 [arXiv:1608.04900] [INSPIRE].

[71] H. Dorn, Holographic entanglement entropy for hollow cones and banana shaped regions, JHEP 06 (2016) 052 [arXiv: 1602.06756] [INSPIRE].

[72] S. Ryu and T. Takayanagi, Holographic derivation of entanglement entropy from AdS/CFT, Phys. Rev. Lett. 96 (2006) 181602 [hep-th/0603001] [INSPIRE].

[73] S. Ryu and T. Takayanagi, Aspects of holographic entanglement entropy, JHEP 08 (2006) 045 [hep-th/0605073] [INSPIRE].

[74] N. Drukker, D.J. Gross and H. Ooguri, Wilson loops and minimal surfaces, Phys. Rev. D 60 (1999) 125006 [hep-th/9904191] [INSPIRE].

[75] H. Dorn, Wilson loops at strong coupling for curved contours with cusps, J. Phys. A 49 (2016) 145402 [arXiv: 1509.00222] [INSPIRE].

[76] H. Dorn, On a new type of divergence for spiky Wilson loops and related entanglement entropies, JHEP 03 (2018) 124 [Erratum ibid. 05 (2018) 108] [arXiv:1801.10367] [INSPIRE].

[77] H. Dorn, On Wilson loops for two touching circles with opposite orientation, J. Phys. A 52 (2019) 095401 [arXiv:1811.00799] [INSPIRE].

[78] H. Dorn, More on Wilson loops for two touching circles, JHEP 07 (2019) 088 [arXiv: 1905.01101] [INSPIRE].

[79] M. Guo and R.C. Myers, private communication.

[80] A.F. Astaneh, G. Gibbons and S.N. Solodukhin, What surface maximizes entanglement entropy?, Phys. Rev. D 90 (2014) 085021 [arXiv:1407.4719] [INSPIRE].

[81] A. Lewkowycz and E. Perlmutter, Universality in the geometric dependence of Rényi entropy, JHEP 01 (2015) 080 [arXiv:1407.8171] [INSPIRE]. 